\title{
Gordon's Conjecture 3: Fourier transforms in the hyperfinite setting
}

PAVOL ZLATOŠ

\begin{abstract}
Using methods of nonstandard analysis and building upon the results of our previous paper in which we proved Gordon's Conjectures 1 and 2 we will show that for any locally compact abelian group $\mathbf{G}$ the Fourier transform $\mathbf{F}: \mathrm{L}^{1}(\mathbf{G}) \rightarrow \mathrm{C}_{0}(\widehat{\mathbf{G}})$, the Fourier-Stieltjes transform $\mathbf{F}: \mathrm{M}(\mathbf{G}) \rightarrow \mathrm{C}_{\mathrm{bu}}(\widehat{\mathbf{G}})$, as well as all the generalized Fourier transforms $\mathbf{F}: \mathrm{L}^{p}(\mathbf{G}) \rightarrow \mathrm{L}^{q}(\widehat{\mathbf{G}})$ for any pair of adjoint exponents $p \in(1,2], q \in[2, \infty)$ can be approximated in a fairly natural way by the discrete Fourier transform $\mathcal{F}: \mathbb{C}^{G} \rightarrow \mathbb{C}^{\widehat{G}}$ on a (hyper)finite abelian group $G$. In particular, we will prove Gordon's Conjecture 3, originally stated for the Fourier-Plancherel transform $\mathbf{F}: \mathrm{L}^{2}(\mathbf{G}) \rightarrow \mathrm{L}^{2}(\widehat{\mathbf{G}})$, and generalize it to all the above mentioned cases. Some standard consequences will be considered, as well.
\end{abstract}

2010 Mathematics Subject Classification 43A25 (primary); 26E35, 28E05, 46S20, 43A10, 43A15, 43A20 (secondary)

Keywords: Locally compact abelian group, Pontryagin-van Kampen duality, Fourier transform, nonstandard analysis, hyperfinite, infinitesimal, approximation

\section{Introduction}

This paper is a direct continuation of our previous work [19], dealing with approximation of locally compact abelian groups (briefly LCA groups) by (hyper)finite abelian groups and using the concepts and methods of nonstandard analysis (NSA). We will assume that the reader is acquainted with the notions and results which were introduced or proved there, and use them freely throughout the present article, frequently without any reference. Some motivational accounts as well as a more detailed outline of the results of the paper follow.

For a finite abelian group $G$ its dual group $\widehat{G}=\operatorname{Hom}(G, \mathbb{T})$, where $\mathbb{T}$ denotes the compact multiplicative group of complex units, is isomorphic (though not canonically) to $G$, the $|G|$-dimensional vector space $\mathbb{C}^{G}$ is endowed with the Hermitian inner product

$$
\langle f, g\rangle_{d}=d \sum_{x \in G} f(x) \overline{g(x)}
$$


where $d>0$ is some normalizing coefficient, the characters $\gamma \in \widehat{G}$ form an orthogonal basis in $\mathbb{C}^{G}$ and the discrete Fourier transform (DFT) $\mathcal{F}: \mathbb{C}^{G} \rightarrow \mathbb{C}^{\widehat{G}}$ is defined as the inner (or scalar) product

$$
\mathcal{F}(f)(\gamma)=\widehat{f}(\gamma)=\langle f, \gamma\rangle_{d}
$$

for $f \in \mathbb{C}^{G}, \gamma \in \widehat{G}$. Once the inner product $\langle\varphi, \psi\rangle_{\widehat{d}}$ on $\mathbb{C}^{\widehat{G}}$ is defined using the adjoint normalizing coefficient $\widehat{d}=(d|G|)^{-1}$, we have the Fourier inversion formula

$$
f=\widehat{d} \sum_{\gamma \in \widehat{G}} \widehat{f}(\gamma) \gamma
$$

and the Plancherel identity

$$
\langle f, g\rangle_{d}=\langle\widehat{f}, \widehat{g}\rangle_{\widehat{d}}
$$

turning the DFT $\mathcal{F}: \mathbb{C}^{G} \rightarrow \mathbb{C}^{\widehat{G}}$ into a linear isometry of unitary spaces.

For a general LCA group $\mathbf{G}$ the picture is not so simple. Its dual group $\widehat{\mathbf{G}}$ consists of all continuous homomorphisms (characters) $\gamma: \mathbf{G} \rightarrow \mathbb{T}$ and the Fourier transform is primarily defined on the Lebesgue space $\mathrm{L}^{1}(\mathbf{G})=\mathrm{L}^{1}(\mathbf{G}, \boldsymbol{m})$, where $\boldsymbol{m}$ is the Haar measure on $\mathbf{G}$, as the bounded linear operator $\mathbf{F}: \mathrm{L}^{1}(\mathbf{G}) \rightarrow \mathrm{C}_{0}(\widehat{\mathbf{G}})$ given by

$$
\mathbf{F}(\boldsymbol{f})(\gamma)=\widehat{\boldsymbol{f}}(\gamma)=\int \boldsymbol{f} \bar{\gamma} \mathrm{d} \boldsymbol{m}
$$

for $\boldsymbol{f} \in \mathrm{L}^{1}(\mathbf{G}), \boldsymbol{\gamma} \in \widehat{\mathbf{G}}$. It can be extended to the so called Fourier-Stieltjes transform F : $\mathrm{M}(\mathbf{G}) \rightarrow \mathrm{C}_{\mathrm{bu}}(\widehat{\mathbf{G}})$ from the Banach space $\mathrm{M}(\mathbf{G}) \supseteq \mathrm{L}^{1}(\mathbf{G})$ of all complex-valued regular Borel measures on $\mathbf{G}$ with finite total variation to the Banach space $\mathrm{C}_{\mathrm{bu}}(\widehat{\mathbf{G}})$ of all bounded uniformly continuous functions $\widehat{\mathbf{G}} \rightarrow \mathbb{C}$, defined by

$$
\mathbf{F}(\boldsymbol{\mu})(\gamma)=\widehat{\mu}(\gamma)=\int \bar{\gamma} \mathrm{d} \boldsymbol{\mu}
$$

for $\boldsymbol{\mu} \in \mathrm{M}(\mathbf{G})$.

Using the density of the intersection $\mathrm{L}^{1}(\mathbf{G}) \cap \mathrm{L}^{p}(\mathbf{G})$ in the Lebesgue space $\mathrm{L}^{p}(\mathbf{G})$ with respect to its norm $\|\cdot\|_{p}$, the Fourier transform can be extended to a bounded linear operator $\mathbf{F}: \mathrm{L}^{p}(\mathbf{G}) \rightarrow \mathrm{L}^{q}(\widehat{\mathbf{G}})$ for $p \in(1,2]$ and the adjoint exponent $q=p /(p-1) \in$ $[2, \infty)$. Under a proper normalization of the Haar measure $\boldsymbol{n}$ on the dual group $\widehat{\mathbf{G}}$ we have the Fourier inversion formula

$$
\boldsymbol{f}=\int \widehat{\boldsymbol{f}}(\gamma) \gamma \mathrm{d} \boldsymbol{n}
$$

(both with respect to the supremum norm $\|\cdot\|_{\infty}$ and the $\mathrm{L}^{p}$-norm $\|\cdot\|_{p}$ ) just for functions $\boldsymbol{f} \in \mathrm{L}^{p}(\mathbf{G}) \cap \mathbf{F}[\mathrm{M}(\widehat{\mathbf{G}})] \subseteq \mathrm{L}^{p}(\mathbf{G}) \cap \mathrm{C}_{\mathrm{bu}}(\mathbf{G})$, with $\mathbf{F}$ denoting the Fourier-Stieltjes transform $\mathrm{M}(\widehat{\mathbf{G}}) \rightarrow \mathrm{C}_{\mathrm{bu}}(\mathbf{G})$. 
For $p=q=2$ we obtain the isometric linear isomorphism $\mathbf{F}: \mathrm{L}^{2}(\mathbf{G}) \rightarrow \mathrm{L}^{2}(\widehat{\mathbf{G}})$ of Hilbert spaces, called the Fourier-Plancherel transform. Then we have the Plancherel identity

$$
\langle\boldsymbol{f}, \boldsymbol{g}\rangle=\int \boldsymbol{f} \overline{\boldsymbol{g}} \mathrm{d} \boldsymbol{m}=\int \widehat{\boldsymbol{f}} \overline{\boldsymbol{g}} \mathrm{d} \boldsymbol{n}=\langle\widehat{\boldsymbol{f}}, \widehat{\boldsymbol{g}}\rangle
$$

(just) for $\boldsymbol{f}, \boldsymbol{g} \in \mathrm{L}^{2}(\mathbf{G})$. Unfortunately, unless $\mathbf{G}$ is compact, $\widehat{\mathbf{G}} \cap \mathrm{L}^{2}(\mathbf{G})=\emptyset$ and the inner product $\langle\gamma, \chi\rangle$ of characters $\gamma, \chi \in \widehat{\mathbf{G}}$ is never defined, so that one can speak of the orthogonal basis formed by the characters at most in a metaphorical sense.

Taking additionally into account that the Fourier transform on finite abelian groups can be computed using the extremely fast and powerful algorithms of the Fast Fourier Transform, there naturally arises the following question:

Given any LCA group $\mathbf{G}$, is there some "universal extension", encompassing all the spaces $\mathrm{L}^{p}(\mathbf{G})$ and $\mathrm{M}(\mathbf{G})$, and a uniform scheme defining the Fourier transform on this extension, covering all the particular Fourier transforms mentioned above, as would be true if $\mathbf{G}$ were finite?

The main goal of this paper is to provide arguments that nonstandard analysis offers a solution to this question which is not only reasonable and satisfactory but also fairly elegant, as well as several additional insights. Our approach is based on some ideas, introduced by Gordon in [6] and further advanced in Gordon [7] and Zlatoš [19]. Namely, in [19] we elaborated concepts and techniques enabling us to approximate simultaneously, with infinitesimal precision on finite elements, any pair consisting of a (Hausdorff) LCA group $\mathbf{G}$ and its dual group $\widehat{\mathbf{G}}$ by a pair of hyperfinite abelian groups $G, \widehat{G}$ and approximating maps $\eta: G \rightarrow{ }^{*} \mathbf{G}, \phi: \widehat{G} \rightarrow{ }^{*} \widehat{\mathbf{G}}$ in such a way that the pairing function $(\boldsymbol{x}, \gamma) \mapsto \gamma(\boldsymbol{x})$ on ${ }^{*} \mathbf{G} \times{ }^{*} \widehat{\mathbf{G}}$ is approximated by the pairing function $(x, \gamma) \mapsto \gamma(x)$ on $G \times \widehat{G}$, and we proved Gordon's Conjectures 1 and 2. From this we derived analogous standard results on arbitrarily precise simultaneous approximation of the pair of LCA groups $\mathbf{G}, \widehat{\mathbf{G}}$ by a finite abelian group $G$ and its dual group $\widehat{G}$, as well as of the pairing function on $\mathbf{G} \times \widehat{\mathbf{G}}$ by the pairing function on $G \times \widehat{G}$.

In the present paper we are going to carry out the third and final of the three steps announced in the introduction to [19], namely to approximate the Fourier transform $\mathbf{F}: \mathrm{L}^{1}(\mathbf{G}) \rightarrow \mathrm{C}_{0}(\widehat{\mathbf{G}})$, the Fourier-Stieltjes transform $\mathbf{F}: \mathrm{M}(\mathbf{G}) \rightarrow \mathrm{C}_{\mathrm{bu}}(\widehat{\mathbf{G}})$, as well as the generalized Fourier transforms $\mathbf{F}: \mathrm{L}^{p}(\mathbf{G}) \rightarrow \mathrm{L}^{q}(\widehat{\mathbf{G}})$, for any pair of adjoint exponents $p \in(1,2], q \in[2, \infty)$, by the hyperfinite dimensional DFT $\mathcal{F}:{ }^{*} \mathbb{C}^{G} \rightarrow{ }^{*} \mathbb{C}^{\widehat{G}}$. In particular, we will prove Gordon's Conjecture 3, originally stated for the FourierPlancherel transform $\mathbf{F}: \mathrm{L}^{2}(\mathbf{G}) \rightarrow \mathrm{L}^{2}(\widehat{\mathbf{G}})$ in Gordon [6, 7], and generalize it to all the cases mentioned above. 
Should we encapsulate the moral of these results in a single sentence, the best we can do seems to be to phrase it as a response to the question formulated in the title of the paper by Epstein [5]:

How well does the finite Fourier transform approximate the Fourier transform?

The response in the abstract of [5] is "very well indeed"; and, in the conclusion: "as well as it possibly could". We aim to convince the reader to agree finally with the following:

\section{Even better than one could ever hope. ${ }^{1}$}

Intuitively, we can unfold the above slogan in the following rather imprecise way: any of the Fourier transforms $\mathrm{L}^{1}(\mathbf{G}) \rightarrow \mathrm{C}_{0}(\widehat{\mathbf{G}}), \mathrm{M}(\mathbf{G}) \rightarrow \mathrm{C}_{\mathrm{bu}}(\widehat{\mathbf{G}}), \mathrm{L}^{p}(\mathbf{G}) \rightarrow \mathrm{L}^{q}(\widehat{\mathbf{G}})$, for adjoint exponents $1<p \leq 2 \leq q<\infty$, can be "arbitrarily well" approximated by the discrete Fourier transform $\mathbb{C}^{G} \rightarrow \mathbb{C}^{\widehat{G}}$, based on some "sufficiently good" simultaneous approximations of $\mathbf{G}, \widehat{\mathbf{G}}$ by finite abelian groups $G, \widehat{G}$, respectively.

The plan of the paper is as follows. Section 1 consists mainly of some preliminary material concerning hyperfinite dimensional representations of some Banach spaces, namely various spaces of continuous functions, the Lebesgue spaces $\mathrm{L}^{p}$ and certain space of complex measures. Thus it is sufficient to highlight some central points from the particular sections of Section 2. All missing notions used here will be defined later in the paper. In the rest of this introduction, $\left(G, G_{0}, G_{\mathrm{f}}\right)$ denotes a condensing IMG group triplet with a hyperfinite abelian ambient group $G$ and a normalizing multiplier $d$, and $\mathbf{G}=G_{\mathrm{f}} / G_{0}$ is its observable trace.

The main result of Section 2.1 is the characterization of internal functions which are liftings (ie, certain kinds of infinitesimally precise approximations) of functions $\boldsymbol{f} \in \mathrm{L}^{p}(\mathbf{G})$ in terms of certain natural continuity condition for the shift $f \mapsto f_{a}$ on the linear space ${ }^{*} \mathbb{C}^{G}$ (cf Theorem 2.1.4). Given an internal norm $N$ on ${ }^{*} \mathbb{C}^{G}$, an internal function $f: G \rightarrow{ }^{*} \mathbb{C}$ is called $S^{\mathrm{N}}$-continuous if $\mathrm{N}\left(f_{a}-f\right) \approx 0$ for any $a \in G_{0}$. Then $S^{p}$-continuous means $S^{\mathrm{N}}$-continuous where $\mathrm{N}$ is the $p$-norm given by $\|f\|_{p}=\left(d \sum_{x \in G}|f(x)|^{p}\right)^{1 / p}$.

A Characterization of Liftings Let $p \in[1, \infty)$ and $f: G \rightarrow{ }^{*} \mathbb{C}$ be an internal function such that $\|f\|_{p}<\infty$ and $\left\|f \cdot 1_{X}\right\|_{p} \approx 0$ for any internal set $X \subseteq G \backslash G_{\mathrm{f}}$. Then $f$ is a lifting of a function $\boldsymbol{f} \in \mathrm{L}^{p}(\mathbf{G})$ if and only if $f$ is $S^{p}$-continuous.

\footnotetext{
${ }^{1}$ In fact, in [5] that question is asked only for the Fourier transform of periodic functions $\mathbb{R} \rightarrow \mathbb{C}$. In our response we include the Fourier transforms on arbitrary LCA groups.
} 
Essentially the same characterization is valid for liftings of functions $\boldsymbol{f} \in \mathrm{C}_{0}(\mathbf{G})$ and the norm $\|f\|_{\infty}=\max _{x \in G}|f(x)|$ (cf Proposition 1.1.1(c)). The sets of all liftings $f$ of functions $f$ from $\mathrm{L}^{p}(\mathbf{G})$ or $\mathrm{C}_{0}(\mathbf{G})$ satisfying the above conditions will be denoted by $\mathcal{L}^{p}\left(G, G_{0}, G_{\mathrm{f}}\right)$ or $\mathcal{C}_{0}\left(G, G_{0}, G_{\mathrm{f}}\right)$, respectively. Additionally, $\mathcal{C}_{\mathrm{bu}}\left(G, G_{0}\right)$ denotes the set of all internal functions $f: G \rightarrow{ }^{*} \mathbb{C}$ satisfying $\|f\|_{\infty}<\infty$ which are $S$-continuous with respect to the equivalence relation corresponding to the monadic subgroup $G_{0} \subseteq G$ and $\mathcal{M}\left(G, G_{0}, G_{\mathrm{f}}\right)$ is the set of all internal functions $f: G \rightarrow{ }^{*} \mathbb{C}$ such that $\|f\|_{1}<\infty$ and $\left\|f \cdot 1_{X}\right\|_{1} \approx 0$ for every internal set $X \subseteq G \backslash G_{\mathrm{f}}$.

The view through the lens of the IMG group triplet $\left(G, G_{0}, G_{f}\right)$ and its dual triplet $\left(\widehat{G}, G_{\mathrm{f}}^{\downarrow}, G_{0}^{\downarrow}\right)$ offers an intuitively appealing explanation of the Smoothness-and-Decay Principle for internal functions $f: G \rightarrow{ }^{*} \mathbb{C}$, based on the formulas for the discrete Fourier transform and its inverse:

$$
\begin{gathered}
\widehat{f}(\gamma)=d \sum_{x \in G} f(x) \bar{\gamma}(x)=d \sum_{x \in G} f(-x) x(\gamma) \\
f(x)=\widehat{d} \sum_{\gamma \in \widehat{G}} \widehat{f}(\gamma) \gamma(x)
\end{gathered}
$$

Intuitively, if $f$ is smooth or continuous in some sense then the contribution of the non- $S$-continuous characters to the expansion of $f$ in the second formula must be somehow negligible. This condition becomes manifest as a kind of quick decay of $\widehat{f}$. The other way around, viewing the elements $x \in G$ as characters of the dual group $\widehat{G}$, the Fourier transform of $f$ can be expressed as their linear combination given by the first formula. If $f$ decays quickly, ie if the values of $f$ on the infinite elements $x \in G \backslash G_{\mathrm{f}}$ are somehow negligible, then the values of its Fourier transform are essentially determined by the values of $f$ on the finite elements $x \in G_{\mathrm{f}}$, which happen to coincide with the $S$-continuous characters of $\widehat{G}$. If neither the coefficients $f(x)$ for $x \in G_{\mathrm{f}}$ are too big then we can reasonably expect $\widehat{f}$ to be smooth or continuous in some sense. This result is proved as our Theorem 2.2.1.

The Smoothness-and-Decay Principle Let N, M be absolute internal norms on the linear spaces ${ }^{*} \mathbb{C}^{G},{ }^{*} \mathbb{C}^{\widehat{G}}$, respectively, such that $\mathrm{N}(f)<\infty$ implies $\mathrm{M}(\widehat{f})<\infty$ for every function $f \in{ }^{*} \mathbb{C}^{G}$. Then for every $f \in{ }^{*} \mathbb{C}^{G}$ the following implications hold:

(a) If $f$ is $S^{\mathrm{N}}$-continuous then $\mathrm{M}\left(\widehat{f} \cdot 1_{\Gamma}\right) \approx 0$ for every internal set $\Gamma \subseteq \widehat{G} \backslash G_{0}^{\downarrow}$.

(b) If $\mathrm{N}(f)<\infty$, and $\mathrm{N}\left(f \cdot 1_{X}\right) \approx 0$ for every internal set $X \subseteq G \backslash G_{\mathrm{f}}$ then $\widehat{f}$ is $S^{\mathrm{M}}$-continuous.

As a consequence of the Hausdorff-Young inequality $\|\widehat{f}\|_{q} \leq\|f\|_{p}$ and the fact that all the $p$-norms are absolute, the Smoothness-and-Decay Principle applies to every pair of norms $\|\cdot\|_{p},\|\cdot\|_{q}$ where $1 \leq p \leq 2 \leq q \leq \infty$ are adjoint exponents. 
The main results of the paper are the following three theorems from Section 2.3, dealing with approximations of the particular classical Fourier transforms by the discrete Fourier transform on the hyperfinite dimensional (HFD) linear space ${ }^{*} \mathbb{C}^{G}$ (cf Theorems 2.3.1-2.3.3).

The HFD Fourier Transform Approximation Theorem Let the internal function $f \in \mathcal{L}^{1}\left(G, G_{0}, G_{\mathrm{f}}\right)$ be a lifting of a function $f \in \mathrm{L}^{1}(\mathbf{G})$. Then the internal function $\mathcal{F}(f)=\widehat{f} \in \mathcal{C}_{0}\left(\widehat{G}, G_{\mathrm{f}}^{\downarrow}, G_{0}^{\downarrow}\right)$ is a lifting of the function $\mathbf{F}(\boldsymbol{f})=\widehat{\boldsymbol{f}} \in \mathrm{C}_{0}(\widehat{\mathbf{G}})$.

The HFD Generalized Fourier Transform Approximation Theorem Let $p \in(1,2]$ and let $q \in[2, \infty)$ be its dual exponent. Let the internal function $f \in \mathcal{L}^{p}\left(G, G_{0}, G_{\mathrm{f}}\right)$ be a lifting of a function $f \in \mathrm{L}^{p}(\mathbf{G})$. Then the internal function $\mathcal{F}(f)=\widehat{f} \in$ $\mathcal{L}^{q}\left(\widehat{G}, G_{\mathrm{f}}^{\downarrow}, G_{0}^{\downarrow}\right)$ is a lifting of the function $\mathbf{F}(\boldsymbol{f})=\widehat{\boldsymbol{f}} \in \mathrm{L}^{q}(\widehat{\mathbf{G}})$.

The special Hilbert space case $p=q=2$ solves Gordon's Conjecture 3 .

The HFD Fourier-Stieltjes Transform Approximation Theorem Let the internal function $g \in \mathcal{M}\left(G, G_{0}, G_{\mathrm{f}}\right)$ be a weak lifting of a complex regular Borel measure $\boldsymbol{\mu} \in \mathrm{M}(\mathbf{G})$. Then the internal function $\mathcal{F}(g)=\widehat{g} \in \mathcal{C}_{\mathrm{bu}}\left(\widehat{G}, G_{\mathrm{f}}^{\perp}\right)$ is a lifting of the function $\mathbf{F}(\boldsymbol{\mu})=\widehat{\boldsymbol{\mu}} \in \mathrm{C}_{\mathrm{bu}}(\widehat{\mathbf{G}})$.

The final section, Section 2.4, addresses some standard analogues of the results of Section 2.3, ie with simultaneous approximations of certain functions or measures on G and their Fourier transforms by functions $f: G \rightarrow \mathbb{C}$ and their discrete Fourier transforms $\widehat{f}: \widehat{G} \rightarrow \mathbb{C}$, respectively, based on simultaneous approximation of the pair of LCA groups $\mathbf{G}, \widehat{\mathbf{G}}$ by a pair of finite abelian groups $G, \widehat{G}$.

Acknowledgement The author acknowledges with thanks the support of his research by the grants no 1/0608/13 and 1/0333/17 of the Slovak Scientific Grant Agency VEGA.

Once again I thank to Zhenya Gordon for steady moral support and encouragement during the almost never ending process of reviewing and revising both my papers on his conjectures.

This paper is dedicated to the memory of Petr Vopěnka (1935-2015). 


\section{Nonstandard counterparts of some Banach spaces}

In this section we briefly review some facts about certain specific nonstandard approaches to Banach spaces, with particular emphasis on some spaces of continuous functions, the Lebesgue spaces $\mathrm{L}^{p}(\mathbf{X})$ for $1 \leq p<\infty$ and the space $\mathbf{M}(\mathbf{X})$ of complex-valued regular Borel measures with finite variation on a Hausdorff locally compact topological space $\mathbf{X}$. Each of these Banach spaces will be associated with a nonstandard counterpart closely related to some hyperfinite dimensional space ${ }^{*} \mathbb{C}^{X}$. The canonical reference for nonstandard Banach space theory is the paper Henson and Moore [9]; additionally, the reader can consult Albeverio, Fenstad, Høegh-Krohn and Lindstrøm [1] and Davis [3].

Throughout the section, $\mathbf{X}$ denotes a Hausdorff locally compact topological space, whose topology is induced by a uniformity $\mathcal{U}$, represented as the observable trace $\mathbf{X} \cong X^{b}$ of a condensing IMG triplet $\left(X, E, X_{\mathrm{f}}\right)$ with a hyperfinite ambient set $X$ by means of a (not necessarily injective) HFI approximation $\eta: X \rightarrow{ }^{*} \mathbf{X}$. Then $X_{\mathrm{f}}=\eta^{-1}\left[\mathrm{Ns}\left({ }^{*} \mathbf{X}\right)\right]$, and we can assume without loss of generality that the equivalence $x \approx y \Leftrightarrow \eta(x) \approx \eta(y)$ holds for all $x, y \in X$ and not just for those in $X_{\mathrm{f}}$. Identifying the observable trace $X^{b}=X_{\mathrm{f}} / E$ with $\mathbf{X} \cong \mathrm{Ns}\left({ }^{*} \mathbf{X}\right) / \approx$ via the homeomorphism $\eta^{b}$, we regard each point $\eta^{b}\left(x^{b}\right)={ }^{\circ} \eta(x) \in \mathbf{X}$ as the observable trace $x^{b}$ of $x \in X_{\mathrm{f}}$ (see Zlatoš [19, Section 1.2]).

The hyperfiniteness of $X$ enables us to represent various Banach spaces of functions $\mathbf{X} \rightarrow \mathbb{R}$ and $\mathbf{X} \rightarrow \mathbb{C}$ by means of the hyperfinite dimensional linear spaces $* \mathbb{R}^{X}$ and $* \mathbb{C}^{X}$ of all internal functions $X \rightarrow{ }^{*} \mathbb{R}$ and $X \rightarrow{ }^{*} \mathbb{C}$, respectively. We will systematically exploit the advantage of such an approach. At the same time, we will focus entirely on spaces of complex functions, leaving the formulation of the real-valued version to the reader.

\subsection{Spaces of continuous functions}

The hyperfinite dimensional (HFD) linear space ${ }^{*} \mathbb{C}^{X}$ admits several internal norms. For any internal norm $\mathrm{N}$ on $* \mathbb{C}^{X}$ we denote by

$$
\begin{aligned}
\mathbb{I}_{\mathrm{N}}{ }^{*} \mathbb{C}^{X} & =\left\{f \in{ }^{*} \mathbb{C}^{X}: \mathrm{N}(f) \approx 0\right\} \\
\mathbb{F}_{\mathrm{N}}{ }^{*} \mathbb{C}^{X} & =\left\{f \in{ }^{*} \mathbb{C}^{X}: \mathrm{N}(f)<\infty\right\}
\end{aligned}
$$

the $\mathbb{F}^{*} \mathbb{C}$-linear subspaces (more precisely, $\mathbb{F}^{*} \mathbb{C}$-submodules) of $* \mathbb{C}^{X}$, consisting of functions which are infinitesimal or finite, respectively, with respect to the norm N. For any "reasonable" norm $\mathrm{N}$, the associated IMG triplet $\left({ }^{*} \mathbb{C}^{X}, \mathbb{I}_{\mathrm{N}}{ }^{*} \mathbb{C}^{X}, \mathbb{F}_{\mathrm{N}}{ }^{*} \mathbb{C}^{X}\right)$, with $\mathbb{I}_{\mathrm{N}}{ }^{*} \mathbb{C}$ standing in place of the indistinguishability equivalence relation

$$
f \approx_{\mathrm{N}} g \Leftrightarrow f-g \in \mathbb{I}_{\mathrm{N}}{ }^{*} \mathbb{C}^{X}
$$


is condensing if and only if $X$ is finite. Its observable trace (nonstandard hull)

$$
\left({ }^{*} \mathbb{C}^{X}\right)_{\mathrm{N}}^{b}=\mathbb{F}_{\mathrm{N}}{ }^{*} \mathbb{C}^{X} / \mathbb{I}_{\mathrm{N}}^{*} \mathbb{C}^{X}
$$

becomes a (standard) Banach space under the norm $\mathrm{N}^{b}$ given by

$$
\mathrm{N}^{b}\left(f_{\mathrm{N}}^{b}\right)={ }^{\circ} \mathrm{N}(f)=\operatorname{st} \mathrm{N}(f)
$$

where $f_{\mathrm{N}}^{b} \in\left({ }^{*} \mathbb{C}^{X}\right)_{\mathrm{N}}^{b}$ denotes the observable trace of the function $f \in \mathbb{F}_{\mathrm{N}}{ }^{*} \mathbb{C}^{X}$ with respect to the norm $\mathrm{N}$. Typically, $\left({ }^{*} \mathbb{C}^{X}\right)_{\mathrm{N}}^{b}$ is nonseparable unless $X$ is finite.

An internal function $f: D \rightarrow{ }^{*} \mathbb{C}$ such that $X_{\mathrm{f}} \subseteq D \subseteq X$ is a triplet morphism $\left(X, E, X_{\mathrm{f}}\right) \rightarrow\left({ }^{*} \mathbb{C}, \mathbb{I}^{*} \mathbb{C}, \mathbb{F}^{*} \mathbb{C}\right)$ if and only if $f$ is $S$-continuous on $X_{\mathrm{f}}$ and $f\left[X_{\mathrm{f}}\right] \subseteq \mathbb{F}^{*} \mathbb{C}$ (see [19, Section 1.2]). Its observable trace is the function $f^{b}: X^{b} \cong \mathbf{X} \rightarrow \mathbb{C}$ given by

$$
f^{b}\left(x^{b}\right)={ }^{\circ} f(x)=\operatorname{st} f(x)
$$

for $x \in X_{\mathrm{f}}$. However, unless $X_{\mathrm{f}}=X$, the monadic equivalence relation on ${ }^{*} \mathbb{C}^{X}$ of infinitesimal nearness on finite elements

$$
f \underset{\tilde{X}_{\mathrm{f}}}{\approx} g \Leftrightarrow\left(\forall x \in X_{\mathrm{f}}\right)(f(x) \approx g(x))
$$

corresponding to the compact-open topology on the space $\mathrm{C}(\mathbf{X})$ of all continuous functions $\mathbf{X} \rightarrow \mathbb{C}$, is not of the form $\approx_{\mathrm{N}}$ for any internal norm $\mathrm{N}$ on ${ }^{*} \mathbb{C}^{X}$.

When dealing with $S$-continuous functions, the maximum norm (or max-norm)

$$
\|f\|_{\infty}=\max _{x \in X}|f(x)|
$$

where $f \in{ }^{*} \mathbb{C}^{X}$, becomes important. Denoting by

$$
\begin{aligned}
\mathbb{I}_{\infty}^{*} \mathbb{C}^{X} & =\left\{f \in{ }^{*} \mathbb{C}^{X}:\|f\|_{\infty} \approx 0\right\} \\
\mathbb{F}_{\infty}{ }^{*} \mathbb{C}^{X} & =\left\{f \in{ }^{*} \mathbb{C}^{X}:\|f\|_{\infty}<\infty\right\}
\end{aligned}
$$

the $\mathbb{F}^{*} \mathbb{C}$-linear subspaces of ${ }^{*} \mathbb{C}^{X}$, consisting of internal functions which are infinitesimal or finite, respectively, with respect to the max-norm we get the IMG triplet $\left({ }^{*} \mathbb{C}^{X}, \mathbb{I}_{\infty}^{*} \mathbb{C}^{X}, \mathbb{F}_{\infty}^{*} \mathbb{C}^{X}\right)$.

We are particularly interested in the Banach spaces $\mathrm{C}_{\mathrm{b}}(\mathbf{X}), \mathrm{C}_{\mathrm{bu}}(\mathbf{X})$, and $\mathrm{C}_{0}(\mathbf{X})$ of all bounded continuous, bounded uniformly continuous, and continuous vanishing at infinity functions $\mathbf{X} \rightarrow \mathbb{C}$, respectively, and also in the (non-Banach) normed linear space $\mathrm{C}_{\mathrm{c}}(\mathbf{X})$ of all continuous functions $\mathbf{X} \rightarrow \mathbb{C}$ with compact support, all with the supremum norm denoted by $\|\cdot\|_{\infty}$, as well. Let us denote by

$$
\begin{aligned}
\mathcal{C}_{\mathrm{b}}\left(X, E, X_{\mathrm{f}}\right) & =\left\{f \in \mathbb{F}_{\infty}{ }^{*} \mathbb{C}^{X}:\left(\forall x, y \in X_{\mathrm{f}}\right)(x \approx y \Rightarrow f(x) \approx f(y))\right\} \\
\mathcal{C}_{\mathrm{bu}}(X, E) & =\left\{f \in \mathbb{F}_{\infty}{ }^{*} \mathbb{C}^{X}:(\forall x, y \in X)(x \approx y \Rightarrow f(x) \approx f(y))\right\} \\
\mathcal{C}_{0}\left(X, E, X_{\mathrm{f}}\right) & =\left\{f \in \mathcal{C}_{\mathrm{b}}\left(X, E, X_{\mathrm{f}}\right):\left(\forall x \in X \backslash X_{\mathrm{f}}\right)(f(x) \approx 0)\right\} \\
\mathcal{C}_{\mathrm{c}}\left(X, E, X_{\mathrm{f}}\right) & =\left\{f \in \mathcal{C}_{\mathrm{b}}\left(X, E, X_{\mathrm{f}}\right):\left(\forall x \in X \backslash X_{\mathrm{f}}\right)(f(x)=0)\right\}
\end{aligned}
$$


their intended nonstandard counterparts. Each $f \in \mathcal{C}_{\mathrm{b}}\left(X, E, X_{\mathrm{f}}\right)$ is an everywhere defined triplet morphism $\left(X, E, X_{\mathrm{f}}\right) \rightarrow\left({ }^{*} \mathbb{C}, \mathbb{I}^{*} \mathbb{C}, \mathbb{F}^{*} \mathbb{C}\right)$ (however there can be also such triplet morphisms not belonging to $\left.\mathcal{C}_{\mathrm{b}}\left(X, E, X_{\mathrm{f}}\right)\right)$. Moreover, we have the obvious inclusions of $\mathbb{F}^{*} \mathbb{C}$-linear subspaces of $* \mathbb{C}^{X}$ :

$$
\mathcal{C}_{\mathrm{c}}\left(X, E, X_{\mathrm{f}}\right)+\mathbb{I}_{\infty}^{*} \mathbb{C}^{X} \subseteq \mathcal{C}_{0}\left(X, E, X_{\mathrm{f}}\right) \subseteq \mathcal{C}_{\mathrm{bu}}(X, E) \subseteq \mathcal{C}_{\mathrm{b}}\left(X, E, X_{\mathrm{f}}\right)
$$

Let us recall from Zlatoš [19] that an internal function $f: X \rightarrow^{*} \mathbb{C}$ is called a lifting of a continuous function $f: \mathbf{X} \rightarrow \mathbb{C}$ if

$$
\boldsymbol{f}\left(\boldsymbol{x}^{b}\right)={ }^{\circ} f(x)
$$

for each $x \in X_{\mathrm{f}}$. It is clear that every lifting $f: X \rightarrow{ }^{*} \mathbb{C}$ of a continuous function $f: \mathbf{X} \rightarrow \mathbb{C}$ is $S$-continuous on $X_{\mathrm{f}}$ and satisfies $f\left[X_{\mathrm{f}}\right] \subseteq \mathbb{F}^{*} \mathbb{C}$, hence, it is a triplet morphism $f:\left(X, \approx, X_{\mathrm{f}}\right) \rightarrow\left({ }^{*} \mathbb{C}, \mathbb{I}^{*} \mathbb{C}, \mathbb{F}^{*} \mathbb{C}\right)$.

Proposition 1.1.1 Let $\boldsymbol{f}: \mathbf{X} \rightarrow \mathbb{C}$ be any continuous function. Then we have:

(a) $\boldsymbol{f} \in \mathrm{C}_{\mathrm{b}}(\mathbf{X})$ if and only if $\boldsymbol{f}$ has a lifting $f \in \mathcal{C}_{\mathrm{b}}\left(X, E, X_{\mathrm{f}}\right)$

(b) $\boldsymbol{f} \in \mathrm{C}_{\mathrm{bu}}(\mathbf{X})$ if and only if $\boldsymbol{f}$ has a lifting $f \in \mathcal{C}_{\mathrm{bu}}(X, E)$

(c) $\boldsymbol{f} \in \mathrm{C}_{0}(\mathbf{X})$ if and only if $\boldsymbol{f}$ has a lifting $f \in \mathcal{C}_{0}\left(X, E, X_{\mathrm{f}}\right)$

(d) $\boldsymbol{f} \in \mathrm{C}_{\mathrm{c}}(\mathbf{X})$ if and only if $\boldsymbol{f}$ has a lifting $f \in \mathcal{C}_{\mathrm{c}}\left(X, E, X_{\mathrm{f}}\right)$

Conversely, every internal function $f \in \mathcal{C}_{\mathrm{b}}\left(X, E, X_{\mathrm{f}}\right)$ is lifting of a function $f \in \mathrm{C}_{\mathrm{b}}(\mathbf{X})$, every internal function $f \in \mathcal{C}_{\mathrm{bu}}(X, E)$ is lifting of a function $f \in \mathrm{C}_{\mathrm{bu}}(\mathbf{X})$, every internal function $f \in \mathcal{C}_{0}\left(X, E, X_{\mathrm{f}}\right)$ is lifting of a function $f \in \mathrm{C}_{0}(\mathbf{X})$, and every internal function $f \in \mathcal{C}_{\mathrm{c}}\left(X, E, X_{\mathrm{f}}\right)$ is lifting of a function $\boldsymbol{f} \in \mathrm{C}_{\mathrm{c}}(\mathbf{X})$.

Proof Let us start with the observation that, under the identification $x^{b}={ }^{\circ} \eta(x)$ for $x \in X_{\mathrm{f}}$, we have $\left({ }^{*} \boldsymbol{f} \circ \eta\right)^{b}=\boldsymbol{f}$ for any continuous function $\boldsymbol{f}: \mathbf{X} \rightarrow \mathbb{C}$. Next, we leave the reader to verify the following easy facts:

$$
\begin{aligned}
\boldsymbol{f} \in \mathrm{C}_{\mathrm{b}}(\mathbf{X}) & \Rightarrow{ }^{*} \boldsymbol{f} \circ \eta \in \mathcal{C}_{\mathrm{b}}\left(X, E, X_{\mathrm{f}}\right) \\
\boldsymbol{f} \in \mathrm{C}_{\mathrm{bu}}(\mathbf{X}) & \Rightarrow{ }^{*} \boldsymbol{f} \circ \eta \in \mathcal{C}_{\mathrm{bu}}(X, E) \\
\boldsymbol{f} \in \mathrm{C}_{0}(\mathbf{X}) & \Rightarrow{ }^{*} \boldsymbol{f} \circ \eta \in \mathcal{C}_{0}\left(X, E, X_{\mathrm{f}}\right) \\
\boldsymbol{f} \in \mathrm{C}_{\mathrm{c}}(\mathbf{X}) & \Rightarrow{ }^{*} \boldsymbol{f} \circ \eta \in \mathcal{C}_{\mathrm{c}}\left(X, E, X_{\mathrm{f}}\right) \\
f \in \mathcal{C}_{\mathrm{b}}\left(X, E, X_{\mathrm{f}}\right) & \Rightarrow f^{b} \in \mathrm{C}_{\mathrm{b}}(\mathbf{X}) \\
f \in \mathcal{C}_{\mathrm{bu}}(X, E) & \Rightarrow f^{b} \in \mathrm{C}_{\mathrm{bu}}(\mathbf{X}) \\
f \in \mathcal{C}_{0}\left(X, E, X_{\mathrm{f}}\right) & \Rightarrow f^{b} \in \mathrm{C}_{0}(\mathbf{X}) \\
f \in \mathcal{C}_{\mathrm{c}}\left(X, E, X_{\mathrm{f}}\right) & \Rightarrow f^{b} \in \mathrm{C}_{\mathrm{c}}(\mathbf{X})
\end{aligned}
$$


for $\boldsymbol{f} \in \mathbb{C}^{\mathbf{X}}, f \in{ }^{*} \mathbb{C}^{X}$. Now, (a), (b), (c) and (d) follow from the first quadruple of implications. (The additional property $E=\{(x, y) \in X \times X: \eta(x) \approx \eta(y)\}$ is needed for the second implication.) The "conversely part" follows from the second quadruple of implications.

We emphasize that in general the observable traces $f^{b}$ and $f_{\infty}^{b}$ of $S$-continuous functions should not be confused. For $f, g \in \mathcal{C}_{0}\left(X, E, X_{\mathrm{f}}\right)$ (and therefore $f, g \in \mathcal{C}_{\mathrm{c}}\left(X, E, X_{\mathrm{f}}\right)$ ) we do have the equivalence:

$$
f^{b}=g^{b} \Leftrightarrow\|f-g\|_{\infty} \approx 0
$$

However, for $f, g \in \mathcal{C}_{\mathrm{bu}}(X, E)$ (and therefore $f, g \in \mathcal{C}_{\mathrm{b}}\left(X, E, X_{\mathrm{f}}\right)$ ) we only have the implication:

$$
\|f-g\|_{\infty} \approx 0 \Rightarrow f^{b}=g^{b}
$$

Unless $X_{\mathrm{f}}$ is dense in $X$, there are functions $f, g \in \mathcal{C}_{\text {bu }}(X, E)$ such that $f^{b}=g^{b}$ but $\|f-g\|_{\infty} \not \approx 0$. Summing up we have the following.

Corollary 1.1.2 The observable trace map $f \mapsto f^{b}$ induces:

(a) a bounded surjective linear mapping of the subspace $\mathcal{C}_{\mathrm{b}}\left(X, E, X_{\mathrm{f}}\right) / \mathbb{I}_{\infty}{ }^{*} \mathbb{C}^{X}$ of the nonstandard hull $\mathbb{F}_{\infty}{ }^{*} \mathbb{C}^{X} / \mathbb{I}_{\infty}{ }^{*} \mathbb{C}^{X}$ onto the Banach space $\mathrm{C}_{\mathrm{b}}(\mathbf{X})$

(b) a bounded surjective linear mapping of the subspace $\mathcal{C}_{\mathrm{bu}}(X, E) / \mathbb{I}_{\infty}{ }^{*} \mathbb{C}^{X}$ of the nonstandard hull $\mathbb{F}_{\infty}{ }^{*} \mathbb{C}^{X} / \mathbb{I}_{\infty}{ }^{*} \mathbb{C}^{X}$ onto the Banach space $\mathrm{C}_{\mathrm{bu}}(\mathbf{X})$

(c) a Banach space isomorphism of the subspace $\mathcal{C}_{0}\left(X, E, X_{\mathrm{f}}\right) / \mathbb{I}_{\infty}{ }^{*} \mathbb{C}^{X}$ of the nonstandard hull $\mathbb{F}_{\infty}{ }^{*} \mathbb{C}^{X} / \mathbb{I}_{\infty}{ }^{*} \mathbb{C}^{X}$ onto the Banach space $\mathrm{C}_{0}(\mathbf{X})$

(d) a normed space isomorphism of the subspace $\mathcal{C}_{\mathrm{c}}\left(X, E, X_{\mathrm{f}}\right) / \mathbb{I}_{\infty}{ }^{*} \mathbb{C}^{X}$ of the nonstandard hull $\mathbb{F}_{\infty}{ }^{*} \mathbb{C}^{X} / \mathbb{I}_{\infty}{ }^{*} \mathbb{C}^{X}$ onto the normed space $\mathrm{C}_{\mathrm{c}}(\mathbf{X})$

\subsection{Lebesgue spaces and spaces of measures}

The natural way of getting functions $\boldsymbol{f}: \mathbf{X} \rightarrow \mathbb{C}$ as observable traces $\boldsymbol{f}=f^{b}$ of internal functions $f: X \rightarrow{ }^{*} \mathbb{C}$ works only under the assumption that $f$ is finite and $S$-continuous on $X_{\mathrm{f}}$. In this way, however, only continuous functions $f: \mathbf{X} \rightarrow \mathbb{C}$ can be obtained. Therefore, many internal functions $f: X \rightarrow{ }^{*} \mathbb{C}$ do not represent classical functions $\mathbf{X} \rightarrow \mathbb{C}$. On the other hand, they can be used to represent various objects of a different nature: measures, distributions, etc. The class of functions $\boldsymbol{f}$ on $\mathbf{X}=X^{b}$ representable that way can be extended to include the Lebesgue spaces $L^{p}(\mathbf{X})$ by relaxing the equality $\boldsymbol{f}\left(x^{b}\right)={ }^{\circ} f(x)$ on $X_{\mathrm{f}}$ to the equality almost everywhere on $X_{\mathrm{f}}$ with respect to some measure. Strictly speaking, the elements of $\mathrm{L}^{p}(\mathbf{X})$ themselves are not genuine functions 
but certain equivalence classes of functions. We are going to make this point more precise. However, instead of functions, we will start with representation of certain complex valued measures

Similarly, as in Zlatoš [19, Section 1.2] every internal function $g: X \rightarrow{ }^{*} \mathbb{C}$ such that $\sum_{x \in X}|g(x)|$ is finite gives rise to the finite complex Loeb measure $\lambda_{g}: \widetilde{\mathcal{P}}(X) \rightarrow \mathbb{C}$, where $\widetilde{\mathcal{P}}(X)$ is a $\sigma$-algebra extending the algebra of internal subsets of $X$, such that

$$
\lambda_{g}(A)={ }^{\circ}\left(\sum_{x \in A} g(x)\right)
$$

for each internal set $A \subseteq X$, and to a complex regular Borel measure $\boldsymbol{\theta}_{g}$ on $\mathbf{X}$ given by

$$
\boldsymbol{\theta}_{g}(\mathbf{Y})=\lambda_{g}\left(\mathbf{Y}^{\sharp}\right)
$$

for Borel $\mathbf{Y} \subseteq \mathbf{X}$. Moreover, $\boldsymbol{\theta}_{g}$ has finite variation:

$$
\left\|\boldsymbol{\theta}_{g}\right\| \leq^{\circ}\left(\sum_{x \in X}|g(x)|\right)=\lambda_{|g|}(X)
$$

Proposition 1.2.1 Every complex regular Borel measure $\boldsymbol{\mu}$ on $\mathbf{X}$ with finite variation has the form $\boldsymbol{\mu}=\boldsymbol{\theta}_{g}$ for some internal function $g: X \rightarrow{ }^{*} \mathbb{C}$, such that

$$
\|\boldsymbol{\mu}\|={ }^{\circ}\left(\sum_{x \in X}|g(x)|\right) \quad \text { and } \quad \sum_{x \in Z}|g(x)| \approx 0
$$

for each internal set $Z \subseteq X \backslash X_{\mathrm{f}}$.

Sketch of proof Essentially the same construction as used to obtain the function $d$ from the nonnegative measure $\boldsymbol{m}$ in [19, Proposition 1.2.6] works for every complex regular Borel measure $\boldsymbol{\mu}$ with finite variation to give the function $g$. One just has to take care that the hyperfinite * Borel partition of $\mathbf{K}$ formed by all the sets of the form $\{\boldsymbol{x} \in \mathbf{K}:(\eta \circ \sigma)(\boldsymbol{x})=\eta(x)\}$, where $x$ runs over some maximal internal set $X_{0} \subseteq \eta^{-1}[\mathbf{U}[\mathbf{K}]]$ such that the restriction $\eta\left\lceil X_{0}\right.$ is injective, satisfies additionally:

$$
\sum_{x \in X_{0}}\left|{ }^{*} \boldsymbol{\mu}(\{\boldsymbol{x} \in \mathbf{K}:(\eta \circ \sigma)(\boldsymbol{x})=\eta(x)\})\right| \leq\|\boldsymbol{\mu}\|
$$

Since this is true for any (standard) $\mathbf{X}$-raster $(\mathbf{K}, \mathbf{U})$ and a finite $(\mathbf{K}, \mathbf{U})$ approximation $\eta: X \rightarrow \mathbf{X}$, the existence of such a ${ }^{*} \mathbf{X}$-raster $(\mathbf{K}, \mathbf{U})$ follows from the saturation assumption. A complete proof of Proposition 1.2.1, following a slightly different chain of arguments, can be found in Ziman and Zlatoš [18, Section 4] (cf also the construction of the functions $g_{\boldsymbol{\mu}}^{\boldsymbol{\pi}}$ in Section 2.4). 
In the rest of this section $d: X \rightarrow{ }^{*} \mathbb{R}$ denotes a fixed internal function such that $d(x)>0$ for each $x \in X$ and $\nu_{d}(A) \in \mathbb{F}^{*} \mathbb{R}$ for each internal set $A \subseteq X_{\mathrm{f}}$, with $\nu_{d}(A) \not \approx 0$ for at least one such $A$. The strict positivity of $d$ entails that the formula

$$
\|f\|_{p, d}=\|f\|_{p}=\left(\sum_{x \in X}|f(x)|^{p} d(x)\right)^{1 / p}
$$

defines an internal norm on the linear space ${ }^{*} \mathbb{C}^{X}$ for each real number $p \geq 1$. In particular, for each internal set $A \subseteq X$ we have

$$
\nu_{d}(A)=\sum_{a \in A} d(a)=\left\|1_{A}\right\|_{1}=\left\|1_{A}\right\|_{p}^{p}
$$

where $1_{A}: X \rightarrow\{0,1\}$ is the indicator or characteristic function of the subset $A$ in $X$. Suppressing $d$ in our notation, we denote by

$$
\begin{aligned}
\mathbb{I}_{p}^{*} \mathbb{C}^{X} & =\left\{f \in{ }^{*} \mathbb{C}^{X}:\|f\|_{p} \approx 0\right\} \\
\mathbb{F}_{p}^{*} \mathbb{C}^{X} & =\left\{f \in{ }^{*} \mathbb{C}^{X}:\|f\|_{p}<\infty\right\}
\end{aligned}
$$

the $\mathbb{F}^{*} \mathbb{C}$-linear subspaces of ${ }^{*} \mathbb{C}^{X}$ consisting of all internal functions which are infinitesimal or finite, respectively, with respect to the $p$-norm $\|\cdot\|_{p}$. Thus we get the IMG triplet $\left({ }^{*} \mathbb{C}^{X}, \mathbb{I}_{p}^{*} \mathbb{C}^{X}, \mathbb{F}_{p}^{*} \mathbb{C}^{X}\right)$.

We also fix the notation $\boldsymbol{m}=\boldsymbol{m}_{d}$ for the nonnegative (and not identically 0 ) regular Borel measure on $\mathbf{X}=X^{b}$ induced by $d$, and $\mathrm{L}^{p}(\mathbf{X})=\mathrm{L}^{p}(\mathbf{X}, \boldsymbol{m})$ for the corresponding Lebesgue spaces with the norms:

$$
\|\boldsymbol{f}\|_{p}=\left(\int|\boldsymbol{f}|^{p} \mathrm{~d} \boldsymbol{m}\right)^{1 / p}
$$

We will relate them to some subspaces of $\mathbb{F}_{p}{ }^{*} \mathbb{C}^{X}$ and of the observable trace (nonstandard hull) $\left({ }^{*} \mathbb{C}^{X}\right)_{p}^{b}=\mathbb{F}_{p}^{*} \mathbb{C}^{X} / \mathbb{I}_{p}^{*} \mathbb{C}^{X}$.

Let $\mathbf{M}(\mathbf{X})$ denote the Banach space of all complex regular Borel measures $\boldsymbol{\mu}$ on $\mathbf{X}$ with finite total variation $\|\boldsymbol{\mu}\|$. According to the Riesz representation theorem, $\mathbf{M}(\mathbf{X})$ is isomorphic to the dual $\mathrm{C}_{0}(\mathbf{X})^{\star}$ of the Banach space $\mathrm{C}_{0}(\mathbf{X})$. By the Radon-Nikodym Theorem, $\mathrm{L}^{1}(\mathbf{X})$ can be identified with the closed subspace of all measures $\boldsymbol{\mu} \in \mathbf{M}(\mathbf{X})$ absolutely continuous with respect to $\boldsymbol{m}$.

This, together with the representation of functions $f \in \mathrm{C}_{0}(\mathbf{X})$ by their liftings which are internal functions $f \in \mathcal{C}_{0}\left(X, E, X_{\mathrm{f}}\right)$ (see Proposition 1.1.1), justifies the following notion. An internal function $g \in{ }^{*} \mathbb{C}^{X}$ is called a weak lifting of the measure $\boldsymbol{\mu} \in \mathbf{M}(\mathbf{X})$, if

$$
\int f^{b} \mathrm{~d} \boldsymbol{\mu}={ }^{\circ}\left(\sum_{x \in X} f(x) g(x) d(x)\right)
$$


for every function $f \in \mathcal{C}_{0}\left(X, E, X_{\mathrm{f}}\right)$. This is obviously equivalent to the condition:

$$
\boldsymbol{\mu}=\boldsymbol{\theta}_{g d}
$$

If $\boldsymbol{\mu}$ is absolutely continuous with respect to $\boldsymbol{m}$ and $\mathrm{d} \boldsymbol{\mu}=\boldsymbol{g} \mathrm{d} \boldsymbol{m}$, where $\boldsymbol{g} \in \mathrm{L}^{1}(\mathbf{X})$, then $g \in{ }^{*} \mathbb{C}^{X}$ is called a weak lifting of $\boldsymbol{g}$ if $g$ is a weak lifting of the measure $\boldsymbol{\mu}$, ie, if and only if

$$
\int f^{b} \boldsymbol{g} \mathrm{d} \boldsymbol{m}={ }^{\circ}\left(\sum_{x \in X} f(x) g(x) d(x)\right)
$$

for every function $f \in \mathcal{C}_{0}\left(X, E, X_{\mathrm{f}}\right)$.

Before formulating what we have just proved, let us introduce some notation and terminology. Showing explicitly the weight function $d$ we denote by $\mathcal{M}\left(X, X_{\mathrm{f}}, d\right)$ the $\mathbb{F} * \mathbb{C}$-linear subspace of ${ }^{*} \mathbb{C}^{X}$ consisting of all internal functions $g: X \rightarrow{ }^{*} \mathbb{C}$ satisfying

$$
\|g\|_{1}<\infty \quad \text { and } \quad\left\|g \cdot 1_{Z}\right\|_{1} \approx 0
$$

for each internal set $Z \subseteq X \backslash X_{\mathrm{f}}$. The last condition simply says that the Loeb measure $\lambda_{|g| d}$ is concentrated on the galaxy of accessible elements $X_{\mathrm{f}}$. Therefore, if $g \in \mathcal{M}\left(X, X_{\mathrm{f}}, d\right)$ then

$$
\int f^{b} \boldsymbol{g} \mathrm{d} \boldsymbol{m}={ }^{\circ}\left(\sum_{x \in X} f(x) g(x) d(x)\right)
$$

holds even for all $f \in \mathcal{C}_{\mathrm{b}}\left(X, E, X_{\mathrm{f}}\right)$. Now, the $\mathbb{F}^{*} \mathbb{C}$-linear subspace $\mathcal{S}\left(X, X_{\mathrm{f}}, d\right)$ of $* \mathbb{C}^{X}$ consists of all functions $g \in \mathcal{M}\left(X, X_{\mathrm{f}}, d\right)$ satisfying the absolute $S$-continuity condition

$$
\nu_{d}(A) \approx 0 \Rightarrow\left\|g \cdot 1_{A}\right\|_{1} \approx 0
$$

for each internal set $A \subseteq X$; functions $g \in \mathcal{S}\left(X, X_{\mathrm{f}}, d\right)$ are called $S$-integrable. Obviously, the last condition is equivalent to absolute continuity of the Loeb measure $\lambda_{|g| d}$ with respect to the Loeb measure $\lambda_{d}$, as well as to absolute continuity of $\boldsymbol{\theta}_{|g| d}$ with respect to $\boldsymbol{m}$. Summing up, we have:

Proposition 1.2.2 (a) Every measure $\boldsymbol{\mu} \in \mathbf{M}(\mathbf{X})$ has a weak lifting $g \in \mathcal{M}\left(X, X_{\mathrm{f}}, d\right)$ such that $\|\boldsymbol{\mu}\|={ }^{\circ}\|g\|_{1}$. Conversely, every function $g \in \mathbb{F}_{1}{ }^{*} \mathbb{C}^{X}$, in particular, every $g \in \mathcal{M}\left(X, X_{\mathrm{f}}, d\right)$, is a weak lifting of the measure $\boldsymbol{\theta}_{g d} \in \mathcal{M}\left(X, X_{\mathrm{f}}, d\right)$.

(b) A measure $\boldsymbol{\mu} \in \mathrm{M}(\mathbf{X})$ has a weak lifting $g \in \mathcal{S}\left(X, X_{\mathrm{f}}, d\right)$ if and only if $\boldsymbol{\mu}$ is absolutely continuous with respect to the measure $\boldsymbol{m}$. Conversely, every function $g \in \mathcal{S}\left(X, X_{\mathrm{f}}, d\right)$ is a weak lifting of the measure $\boldsymbol{\theta}_{g d} \in \mathcal{M}\left(X, X_{\mathrm{f}}, d\right)$ (which is absolutely continuous with respect to $\boldsymbol{m}$ ). 
Now, there arises a natural question, namely what is the relation between the weak lifting $g \in \mathcal{S}\left(X, X_{\mathrm{f}}, d\right)$ of an absolutely continuous measure $\boldsymbol{\mu} \in \mathbf{M}(\mathbf{X})$ and the (unique) function $\boldsymbol{g} \in \mathrm{L}^{1}(\mathbf{X})$ such that $\mathrm{d} \boldsymbol{\mu}=\boldsymbol{g} \mathrm{d} \boldsymbol{m}$, ie, between $\boldsymbol{g}$ and its weak lifting $g$. Unless $\boldsymbol{g}$ is continuous, we cannot have $\boldsymbol{g}=g^{b}$, and, unless $g$ is $S$-continuous on $X_{\mathrm{f}}$, the formula for $g^{b}$ doesn't make sense. Nevertheless, we can still generalize the original notion of lifting of continuous functions in the following sense. An internal function $g: X \rightarrow{ }^{*} \mathbb{C}$ is called a lifting of a function $\boldsymbol{g}: \mathbf{X} \rightarrow \mathbb{C}$ (with respect to the weight function $d$ ) if the equality

$$
\boldsymbol{g}\left(x^{b}\right)={ }^{\circ} g(x)
$$

holds for almost all $x \in X_{\mathrm{f}}$ with respect to the Loeb measure $\lambda_{d}$. As the function $\boldsymbol{g} \in \mathrm{L}^{1}(\mathbf{X})$ is only determined up to the equality almost everywhere with respect to the measure $\boldsymbol{m}=\boldsymbol{m}_{d}$, this is the best one can expect.

Proposition 1.2.3 (a) Let $\boldsymbol{g} \in \mathrm{L}^{1}(\mathbf{X})$ and $g \in \mathbb{F}_{1}{ }^{*} \mathbb{C}^{X}$. Then $g$ is a weak lifting of $\boldsymbol{g}$ if and only if $g$ is a lifting of $\mathbf{g}$.

(b) Let $\mathbf{g}: \mathbf{X} \rightarrow \mathbb{C}$. Then the following conditions are equivalent:

(i) $\boldsymbol{g} \in \mathrm{L}^{1}(\mathbf{X})$

(ii) $\mathbf{g}$ has a weak lifting $g \in \mathcal{S}\left(X, X_{\mathrm{f}}, d\right)$

(iii) $g$ has a lifting $g \in \mathcal{S}\left(X, X_{\mathrm{f}}, d\right)$

Sketch of proof (a) If $g \in \mathbb{F}_{1}{ }^{*} \mathbb{C}^{X}$ is a lifting of $\boldsymbol{g}$ then it is obviously a weak lifting of $\boldsymbol{g}$. The reverse implication follows from Proposition 1.1.1(c) and the uniqueness part of the Radon-Nikodym Theorem.

(b) As the implications (iii) $\Rightarrow$ (ii) $\Rightarrow$ (i) are obvious, it suffices to prove (i) $\Rightarrow$ (iii). To this end denote by $\boldsymbol{\mu} \in \mathrm{M}(\mathbf{X})$ the measure satisfying $\mathrm{d} \boldsymbol{\mu}=\boldsymbol{g} \mathrm{d} \boldsymbol{m}$ and by $\widetilde{g} \in{ }^{*} \mathbb{C}^{X}$ the internal function guaranteed to $\boldsymbol{\mu}$ in Proposition 1.2.1. Then the function $g=\widetilde{g} / d$ has all the required properties and ${ }^{\circ} g(x)=\boldsymbol{g}\left(x^{b}\right)$ for $\lambda_{d}$-almost all $x \in X_{\mathrm{f}}$, again due to the uniqueness part of the Radon-Nikodym Theorem.

Remark It is worthwhile to notice that, in the notation from the proof of Zlatoš [19, Proposition 1.2.6], for a "typical" $x \in X_{\mathrm{f}}$ we have

$$
g(x) \approx \frac{{ }^{*} \boldsymbol{\mu}(\{\boldsymbol{x} \in \mathbf{K}:(\eta \circ \sigma)(\boldsymbol{x})=\eta(x)\})}{{ }^{*} \boldsymbol{m}(\{\boldsymbol{x} \in \mathbf{K}:(\eta \circ \sigma)(\boldsymbol{x})=\eta(x)\})}
$$

where the right hand term is the mean value of the function ${ }^{*} \boldsymbol{g}={ }^{*}(\mathrm{~d} \boldsymbol{\mu} / \mathrm{d} \boldsymbol{m})$ on the set $\{\boldsymbol{x} \in \mathbf{K}:(\eta \circ \sigma)(\boldsymbol{x})=\eta(x)\} \subseteq \mathbf{U}[\eta(x)]$. This is in accord with the intuition that the Radon-Nikodym derivative $\boldsymbol{g}(\boldsymbol{x})=(\mathrm{d} \boldsymbol{\mu} / \mathrm{d} \boldsymbol{m})(\boldsymbol{x})$ is the ratio $\boldsymbol{\mu}(\mathbf{V}) / \boldsymbol{m}(\mathbf{V})$ of measures of some "infinitesimal neighborhood" $\mathbf{V}$ of the point $\boldsymbol{x} \in \mathbf{X}$. 
Unfortunately, not every $S$-integrable function is a lifting of some function $g \in \mathrm{L}^{1}(\mathbf{X})$. For instance, every function $g \in \mathbb{F}_{\infty}{ }^{*} \mathbb{C}^{X}$ with internal support

$$
\operatorname{supp} g=\{x \in X: g(x) \neq 0\}
$$

contained in $X_{\mathrm{f}}$ is $S$-integrable; however, unless $E$ is internal, such a function need not be lifting of any function $\boldsymbol{g} \in \mathrm{L}^{1}(\mathbf{X})$. One can naturally expect that, in order to lift a function $\boldsymbol{g} \in \mathrm{L}^{1}(\mathbf{X})$, the function $g \in{ }^{*} \mathbb{C}^{X}$ has to display some "reasonable amount" of continuity, which is not clear for the moment. This leads us to define the external subspace $\mathcal{L}^{1}\left(X, E, X_{\mathrm{f}}\right) \subseteq{ }^{*} \mathbb{C}^{X}$ as the space of all internal functions $g \in \mathcal{M}\left(X, X_{\mathrm{f}}, d\right)$ which are liftings of functions $\boldsymbol{g} \in \mathrm{L}^{1}(\mathbf{X})$. Further we put

$$
\begin{aligned}
\mathcal{M}^{p}\left(X, X_{\mathrm{f}}, d\right) & =\left\{f \in{ }^{*} \mathbb{C}^{X}:|f|^{p} \in \mathcal{M}\left(X, X_{\mathrm{f}}, d\right)\right\} \\
\mathcal{S}^{p}\left(X, X_{\mathrm{f}}, d\right) & =\left\{f \in{ }^{*} \mathbb{C}^{X}:|f|^{p} \in \mathcal{S}\left(X, X_{\mathrm{f}}, d\right)\right\} \\
\mathcal{L}^{p}\left(X, E, X_{\mathrm{f}}\right) & =\left\{f \in{ }^{*} \mathbb{C}^{X}:|f|^{p} \in \mathcal{L}^{1}\left(X, E, X_{\mathrm{f}}\right)\right\}
\end{aligned}
$$

for $1 \leq p<\infty$. Obviously, all the functions in $\mathcal{L}^{1}\left(X, E, X_{\mathrm{f}}\right)$ are $S$-integrable, hence $\mathcal{L}^{p}\left(X, E, X_{\mathrm{f}}\right) \subseteq \mathcal{S}^{p}\left(X, X_{\mathrm{f}}, d\right)$, and the subspaces $\mathcal{L}^{p}\left(X, E, X_{\mathrm{f}}\right)$ are formed by the liftings of functions $\boldsymbol{g} \in \mathrm{L}^{p}(\mathbf{X})$.

Here we do not present a more explicit description of the spaces $\mathcal{L}^{p}\left(X, E, X_{\mathrm{f}}\right)$. However, in case that $\left(G, G_{0}, G_{\mathrm{f}}\right)$ is a condensing IMG group triplet with a hyperfinite abelian ambient group $G$ and $\boldsymbol{m}_{d}$ is the Haar measure on its observable trace $\mathbf{G}=G_{\mathrm{f}} / G_{0}$, we will give a characterization of functions in $\mathcal{L}^{p}\left(G, G_{0}, G_{\mathrm{f}}\right)$ as those belonging to $\mathcal{M}^{p}\left(G, G_{\mathrm{f}}, d\right)$ and satisfying a certain natural continuity condition (see Theorem 2.1.4).

From the definition of $\mathcal{L}^{p}\left(X, E, X_{\mathrm{f}}\right)$ and the last Proposition we readily obtain the following result, justifying our notation.

Proposition 1.2.4 Let $1 \leq p<\infty$. Then the Lebesgue space $\mathrm{L}^{p}(\mathbf{X})$ is isomorphic to the closed subspace $\mathcal{L}^{p}\left(X, E, X_{\mathrm{f}}\right) / \mathbb{I}_{p}{ }^{*} \mathbb{C}^{X}$ of the nonstandard hull $\mathbb{F}_{p}^{*} \mathbb{C}^{X} / \mathbb{I}_{p}^{*} \mathbb{C}^{X}$.

Remark Though, in general, $\mathcal{L}^{p}\left(X, E, X_{\mathrm{f}}\right)$ is a proper subspace of $\mathcal{S}^{p}\left(X, X_{\mathrm{f}}, d\right)$, from 1.2.2 and 1.2.3 it follows that $\mathcal{L}^{p}\left(X, E, X_{\mathrm{f}}\right)$ is dense in $\mathcal{S}^{p}\left(X, X_{\mathrm{f}}, d\right)$ with respect to a natural weak topology which we need not describe precisely here.

\section{The Fourier Transform on hyperfinite abelian groups}

This section addresses our main topics, namely (i) the analysis of the discrete Fourier transform on some subspaces of the hyperfinite dimensional linear space ${ }^{*} \mathbb{C}^{G}$, arising 
from a condensing IMG group triplet $\left(G, G_{0}, G_{\mathrm{f}}\right)$ with hyperfinite abelian ambient group $G$, and (ii) its application to Fourier transforms on various spaces of functions $\boldsymbol{f}: \mathbf{G} \rightarrow \mathbb{C}$ defined on its observable trace, the Hausdorff LCA group $\mathbf{G}=G_{\mathrm{f}} / G_{0}$. In particular, we will formulate and prove a generalization of the third of Gordon's Conjectures to approximations of Fourier transforms $\mathrm{L}^{1}(\mathbf{G}) \rightarrow \mathrm{C}_{0}(\widehat{\mathbf{G}}), \mathrm{M}(\mathbf{G}) \rightarrow \mathrm{C}_{\mathrm{bu}}(\widehat{\mathbf{G}})$ and $\mathrm{L}^{p}(\mathbf{G}) \rightarrow \mathrm{L}^{q}(\widehat{\mathbf{G}})$, for adjoint exponents $1<p \leq 2 \leq q<\infty$, by the discrete Fourier transform $* \mathbb{C}^{G} \rightarrow{ }^{*} \mathbb{C}^{\widehat{G}}$.

Throughout the first three parts of Section 2, $\left(G, G_{0}, G_{\mathrm{f}}\right)$ denotes a condensing IMG group triplet with hyperfinite abelian ambient group $G$ in a sufficiently saturated nonstandard universe. Its observable trace is denoted by $\mathbf{G}=G^{b}=G_{\mathrm{f}} / G_{0}$, and it is a Hausdorff LCA group. Further, $d$ denotes a normalizing coefficient for this triplet and all the norms $\|\cdot\|_{p}$, for $1 \leq p<\infty$, on the linear space ${ }^{*} \mathbb{C}^{G}$ are defined using $d$. Similarly, $\boldsymbol{m}=\boldsymbol{m}_{d}$ denotes the Haar measure on $\mathbf{G}$ obtained by pushing down the Loeb measure $\lambda_{d}$ on $G$ and the norms $\|\cdot\|_{p}$ on the Lebesgue spaces $L^{p}(\mathbf{G})$ are defined via $\boldsymbol{m}$. An analogous convention is adopted for the dual group $\widehat{\mathbf{G}}$ and its Haar measure $\boldsymbol{n}=\boldsymbol{m}_{\widehat{d}}$ obtained from the normalizing multiplier $\widehat{d}=(d|G|)^{-1}$ for the dual triplet $\left(\widehat{G}, G_{\mathrm{f}}^{\downarrow}, G_{0}^{\downarrow}\right)$ (cf Zlatoš [19, Sections 2.2, 2.3]).

\subsection{A characterization of liftings}

In Section 1.2 we noted that for a locally compact Hausdorff space $\mathbf{X}$, represented as the observable trace $\mathbf{X} \cong X^{b}=X_{\mathrm{f}} / E$ of an IMG triplet $\left(X, E, X_{\mathrm{f}}\right)$ with hyperfinite $X$, not every $S$-integrable function $f \in{ }^{*} \mathbb{C}^{X}$ is lifting of some function $f \in \mathrm{L}^{1}(\mathbf{X})$, but were not able to describe these liftings more closely. For Hausdorff LCA groups, however, we can give an intuitively appealing characterization of such liftings in terms of a certain continuity condition.

Let $\mathrm{N}$ be an arbitrary internal norm on the vector space ${ }^{*} \mathbb{C}^{G}$. An internal function $f: G \rightarrow{ }^{*} \mathbb{C}$ is called $S$-continuous with respect to the norm $\mathrm{N}$ (or, briefly, $S^{\mathrm{N}}-$ continuous) if

$$
\mathrm{N}\left(f_{a}-f\right) \approx 0
$$

for each $a \in G_{0}$. (Here $f_{a}(x)=f(x-a)$ for $a, x \in G$.) In case of the $p$-norms we speak about $S^{p}$-continuous functions. In particular, $S^{\infty}$-continuity coincides with the usual notion of $S$-continuity.

Let us recall from $\left[19\right.$, Section 2.1] that a norm $N$ on ${ }^{*} \mathbb{C}^{G}$ is called translation invariant if $\mathrm{N}\left(f_{a}\right)=\mathrm{N}(f)$ for any $f \in{ }^{*} \mathbb{C}^{G}, a \in G$. The following lemma is obvious, once we 
realize that

$$
(f * g)_{a}-f * g=\left(f_{a}-f\right) * g \quad \text { and } \quad \mathrm{N}(f * g) \leq \mathrm{N}(f)\|g\|_{1}
$$

for any functions $f, g \in{ }^{*} \mathbb{C}^{G}, a \in G$ and internal translation invariant norm N.

Lemma 2.1.1 Let $\mathrm{N}$ be an internal translation invariant norm on ${ }^{*} \mathbb{C}^{G}$ and $f, g \in{ }^{*} \mathbb{C}^{G}$. If $f$ is $S^{\mathrm{N}}$-continuous and $\|g\|_{1}<\infty$ then $f * g$ is $S^{\mathrm{N}}$-continuous as well.

In analyzing the structure of $S^{\mathrm{N}}$-continuous functions we will make use of a family of internal functions akin to the family $h_{\varrho}$ defined in the proof of [19, Proposition 2.3.3]. For any gauge $\varrho \in \mathcal{V}$ (see the text preceding [19, Proposition 1.3.1]) and $r>0$ we put:

$$
\vartheta_{\varrho r}=\left\|h_{\varrho}\right\|_{1}^{-1} h_{\varrho}
$$

Then each of the functions $\vartheta_{\varrho}$ is $S$-continuous, even, nonnegative and satisfies both $\left\|\vartheta_{\varrho r}\right\|_{1}=1$ and $\left\|\vartheta_{\varrho r}\right\|_{\infty}<\infty$. Moreover, $G_{0} \subseteq \operatorname{supp} \vartheta_{\varrho r} \subseteq B_{\varrho}(r)$, thus, in particular, $\vartheta_{\varrho} \in \mathcal{C}_{\mathrm{c}}\left(G, G_{0}, G_{\mathrm{f}}\right)$.

The family of internal functions $\vartheta_{\varrho}$ behaves like an approximate unit for the operation of convolution on the set of all $S^{\mathrm{N}}$-continuous functions in the sense of Hewitt and Ross $[10,11]$. The precise formulation follows.

Lemma 2.1.2 Let $\mathrm{N}$ be any internal norm on ${ }^{*} \mathbb{C}^{G}$. Then for every $S^{\mathrm{N}}$-continuous function $f \in{ }^{*} \mathbb{C}^{G}$ the system of functions $\vartheta_{\varrho r} * f$, where $\varrho \in \mathcal{V}, 0<r \in \mathbb{R}$, converges to the function $f$ with respect to the norm $\mathrm{N}$ in the following sense: for each (standard) $\varepsilon>0$ there is an internal set $Q$ such that $G_{0} \subseteq Q \subseteq G$ and for any $\varrho, r$ the inclusion $B_{\varrho}(r) \subseteq Q$ implies $\mathrm{N}\left(f-\vartheta_{\varrho r} * f\right) \leq \varepsilon$. Consequently, if $\varrho \in{ }^{*} \mathcal{V}, 0<r \in{ }^{*} \mathbb{R}$ are such that $B_{\varrho}(r) \subseteq G_{0}$, then $\mathrm{N}\left(f-\vartheta_{\varrho} * f\right) \approx 0$.

Let us remark that, for each internal set $Q \supseteq G_{0}$, there are indeed $\varrho \in \mathcal{V}$ and $r>0$ such that $B_{\varrho}(r) \subseteq Q$, hence the situation described in the lemma is not merely hypothetical.

Proof The $S^{\mathrm{N}}$-continuity of $f$ means, in standard terms, that for each $\varepsilon>0$ there is an internal set $Q \supseteq G_{0}$ such that $\mathrm{N}\left(f_{a}-f\right) \leq \varepsilon$, for $a \in Q$. Assume that $B_{\varrho}(r) \subseteq Q$. Since $\vartheta_{\varrho}$ is nonnegative, $\left\|\vartheta_{\varrho r}\right\|_{1}=1$ and $\operatorname{supp} \vartheta_{\varrho r} \subseteq B_{\varrho}(r)$, we have

$$
f-\vartheta_{\varrho r} * f=\left\|\vartheta_{\varrho r}\right\|_{1} f-d \sum_{a \in G} \vartheta_{\varrho}(a) f_{a}=d \sum_{a \in B_{\varrho}(r)} \vartheta_{\varrho r}(a)\left(f-f_{a}\right)
$$

hence

$$
\mathrm{N}\left(f-\vartheta_{\varrho} r * f\right) \leq d \sum_{a \in B_{\varrho}(r)}\left|\vartheta_{\varrho r}(a)\right| \mathrm{N}\left(f-f_{a}\right) \leq\left\|\vartheta_{\varrho r}\right\|_{1} \max _{a \in B_{\varrho}(r)} \mathrm{N}\left(f-f_{a}\right) \leq \varepsilon
$$


since $\mathrm{N}\left(f-f_{a}\right) \leq \varepsilon$ for $a \in B_{\varrho}(r) \subseteq Q$.

The last sentence of Lemma 2.1.2 is an immediate consequence of the standard statement just proved.

The last of our lemmas deals with a density condition for certain $S^{\mathrm{N}}$-continuous functions. To this end denote by $\mathcal{C}_{\mathrm{c}}^{\mathrm{N}, 1}\left(G, G_{0}, G_{\mathrm{f}}\right)$ the $\mathbb{F}^{*} \mathbb{C}$-linear subspace of the internal space ${ }^{*} \mathbb{C}^{G}$ consisting of all $S^{\mathrm{N}}$-continuous functions $f \in{ }^{*} \mathbb{C}^{G}$ satisfying $\mathrm{N}(f)<\infty,\|f\|_{1}<\infty$ and $\operatorname{supp} f \subseteq G_{\mathrm{f}}$. If $\mathrm{N}$ is the $p$-norm $\|\cdot\|_{p}$ we write $\mathcal{C}_{\mathrm{c}}^{p, 1}\left(G, G_{0}, G_{\mathrm{f}}\right)$.

Lemma 2.1.3 Let $\mathrm{N}$ be any internal norm on ${ }^{*} \mathbb{C}^{G}$. If the subspace $\mathcal{C}_{\mathrm{c}}\left(G, G_{0}, G_{\mathrm{f}}\right)$ is contained in the subspace $\mathcal{C}_{\mathrm{c}}^{\mathrm{N}, 1}\left(G, G_{0}, G_{\mathrm{f}}\right)$ then $\mathcal{C}_{\mathrm{c}}\left(G, G_{0}, G_{\mathrm{f}}\right)$ is dense in $\mathcal{C}_{\mathrm{c}}^{\mathrm{N}, 1}\left(G, G_{0}, G_{\mathrm{f}}\right)$ with respect to the norm $\mathrm{N}$.

Proof Taking any function $f \in \mathcal{C}_{\mathrm{c}}^{\mathrm{N}, 1}\left(G, G_{0}, G_{\mathrm{f}}\right)$, we know that the system of functions $\vartheta_{\varrho r} * f$, where $\varrho \in \mathcal{V}, r>0$, converges to $f$ with respect to $\mathrm{N}$ in the sense of Lemma 2.1.2. It remains to show that $\vartheta * f \in \mathcal{C}_{\mathrm{c}}\left(G, G_{0}, G_{\mathrm{f}}\right)$ for each such a function $\vartheta=\vartheta_{\varrho r}$.

Clearly,

$$
\operatorname{supp}(\vartheta * f) \subseteq \operatorname{supp} \vartheta+\operatorname{supp} f \subseteq G_{\mathrm{f}}
$$

and, according to the fact that the max-norm $\|\cdot\|_{\infty}$ is translation invariant:

$$
\|\vartheta * f\|_{\infty} \leq\|\vartheta\|_{\infty}\|f\|_{1}<\infty
$$

For the same reason, as the function $\vartheta$ is $S$-continuous and $\|f\|_{1}<\infty$, the $S$-continuity of the function $\vartheta * f$ follows from Lemma 2.1.1 applied to the norm $\|\cdot\|_{\infty}$.

As all the internal norms $\|\cdot\|_{p}, 1 \leq p \leq \infty$, on ${ }^{*} \mathbb{C}^{G}$ are translation invariant and satisfy the inclusion $\mathcal{C}_{\mathrm{c}}\left(G, G_{0}, G_{\mathrm{f}}\right) \subseteq \mathcal{C}_{\mathrm{c}}^{p, 1}\left(G, G_{0}, G_{\mathrm{f}}\right)$, Lemmas 2.1.1-2.1.3 apply to them in particular.

Further, let us denote by

$$
\mathcal{M}\left(G, G_{0}, G_{\mathrm{f}}\right)=\left\{f \in{ }^{*} \mathbb{C}^{G}:\|f\|_{1}<\infty \&\left(\forall^{\mathrm{int}} Z \subseteq G \backslash G_{\mathrm{f}}\right)\left(\left\|f \cdot 1_{Z}\right\|_{1} \approx 0\right)\right\}
$$

the $\mathbb{F}^{*} \mathbb{C}$-linear subspace of ${ }^{*} \mathbb{C}^{G}$, formerly denoted as $\mathcal{M}\left(G, G_{\mathrm{f}}, d\right)$. Displaying $G_{0}$ and hiding $d$ is unambiguous since $\mathcal{M}\left(G, G_{\mathrm{f}}, d\right)=\mathcal{M}\left(G, G_{\mathrm{f}}, d^{\prime}\right)$ for any pair of normalizing multipliers $d, d^{\prime}$ of the triplet $\left(G, G_{0}, G_{\mathrm{f}}\right)$. The relation of the subspace $\mathcal{M}\left(G, G_{0}, G_{\mathrm{f}}\right)=\mathcal{M}\left(G, G_{\mathrm{f}}, d\right)$ to the Banach space $\mathrm{M}(\mathbf{G})$ of all complex regular Borel 
measures with finite variation on $\mathbf{G}$ via weak liftings is described in Proposition 1.2.2. As in Section 1.2 we put

$$
\mathcal{M}^{p}\left(G, G_{0}, G_{\mathrm{f}}\right)=\left\{f \in{ }^{*} \mathbb{C}^{G}:|f|^{p} \in \mathcal{M}\left(G, G_{0}, G_{\mathrm{f}}\right)\right\}
$$

for $1 \leq p<\infty$.

We are going to characterize the subspaces $\mathcal{L}^{p}\left(G, G_{0}, G_{\mathrm{f}}\right)$ of the internal linear space ${ }^{*} \mathbb{C}^{G}$ formed by liftings $f \in \mathcal{M}^{p}\left(G, G_{0}, G_{\mathrm{f}}\right)$ of functions $f \in \mathrm{L}^{p}(\mathbf{G})$ for $1 \leq p<\infty$. The following theorem resembles an early theorem by Rudin [15], characterizing measures $\boldsymbol{\mu} \in \mathrm{M}(\mathbf{G})$ arising from functions $\boldsymbol{f} \in \mathrm{L}^{p}(\mathbf{G})$ (ie, $\mathrm{d} \boldsymbol{\mu}=\boldsymbol{f} \mathrm{d} \boldsymbol{m}$ ) as those for which the shift $\boldsymbol{a} \mapsto \boldsymbol{\mu}_{\boldsymbol{a}}(\mathbf{B})=\boldsymbol{\mu}(\mathbf{B}-\boldsymbol{a})$ is a continuous function $\mathbf{G} \rightarrow \mathbb{C}$ for any Borel set $\mathbf{B} \subseteq \mathbf{G}$.

Theorem 2.1.4 (A Characterization of Liftings) Let $1 \leq p<\infty$ and $f$ be an internal function belonging to $\mathcal{M}^{p}\left(G, G_{0}, G_{\mathrm{f}}\right)$. Then $f \in \mathcal{L}^{p}\left(G, G_{0}, G_{\mathrm{f}}\right)$ if and only if $f$ is $S^{p}$-continuous.

Proof For brevity let us denote:

$$
\mathcal{C M}^{p}\left(G, G_{0}, G_{\mathrm{f}}\right)=\left\{f \in \mathcal{M}^{p}\left(G, G_{0}, G_{\mathrm{f}}\right): f \text { is } S^{p} \text {-continuous }\right\}
$$

Then we are to prove that $\mathcal{L}^{p}\left(G, G_{0}, G_{\mathrm{f}}\right)=\mathcal{C M}^{p}\left(G, G_{0}, G_{\mathrm{f}}\right)$. Clearly, they both are subspaces of the internal vector space ${ }^{*} \mathbb{C}^{G}$ and contain the subspace $\mathcal{C}_{\mathrm{c}}\left(G, G_{0}, G_{\mathrm{f}}\right)$. We divide the proof into three simpler Claims. Putting them together, the Theorem easily follows.

Claim $1 \mathcal{L}^{p}\left(G, G_{0}, G_{\mathrm{f}}\right)$ is closed in ${ }^{*} \mathbb{C}^{G}$ with respect to the norm $\|\cdot\|_{p}$.

This is almost obvious. If $\left(f_{n}\right)_{n \in \mathbb{N}}$ is a sequence in $\mathcal{L}^{p}\left(G, G_{0}, G_{\mathrm{f}}\right)$ converging to a function $f \in{ }^{*} \mathbb{C}^{G}$ and each $f_{n}$ is a lifting of some $\boldsymbol{f}_{n} \in \mathrm{L}^{p}(\mathbf{G})$ then the sequence $\left(\boldsymbol{f}_{n}\right)_{n \in \mathbb{N}}$ satisfies the Bolzano-Cauchy condition, hence it converges to a function $f \in \mathrm{L}^{p}(\mathbf{G})$. It is routine to check that $f$ is a lifting of $f$, ie $f \in \mathcal{L}^{p}\left(G, G_{0}, G_{\mathrm{f}}\right)$.

Claim $2 \mathcal{L}^{p}\left(G, G_{0}, G_{\mathrm{f}}\right) \subseteq \mathcal{C M}^{p}\left(G, G_{0}, G_{\mathrm{f}}\right)$

It suffices to show that each lifting $f$ of a function $f \in \mathrm{L}^{p}(\mathbf{G})$ is $S^{p}$-continuous. It is known that the shift $\boldsymbol{a} \mapsto \boldsymbol{f}_{\boldsymbol{a}}$ is a uniformly continuous mapping $\mathbf{G} \rightarrow \mathrm{L}^{p}(\mathbf{G})$ (see Pedersen [13] or Rudin [16]). Translating this condition into the language of infinitesimals one readily obtains the $S^{p}$-continuity of $f$.

Claim $3 \mathcal{C}_{\mathrm{c}}\left(G, G_{0}, G_{\mathrm{f}}\right)$ is dense in $\mathcal{C M}^{p}\left(G, G_{0}, G_{\mathrm{f}}\right)$ with respect to the norm $\|\cdot\|_{p}$.

According to Lemma 2.1.3 it is enough to show that the subspace $\mathcal{C}_{\mathrm{c}}^{p, 1}\left(G, G_{0}, G_{\mathrm{f}}\right)$ is dense in $\mathcal{C M}^{p}\left(G, G_{0}, G_{\mathrm{f}}\right)$ with respect to $\|\cdot\|_{p}$. Let $f \in \mathcal{C M}^{p}\left(G, G_{0}, G_{\mathrm{f}}\right)$. Then 
$\left\|f \cdot 1_{G \backslash Z}\right\|_{p} \approx 0$ for any internal set $Z \subseteq G \backslash G_{\mathrm{f}}$. Due to saturation, there exists a sequence of internal sets $\left(A_{n}\right)_{n \in \mathbb{N}}$ such that $G_{0} \subseteq A_{n} \subseteq G_{\mathrm{f}}$,

$$
\left\|f \cdot 1_{G \backslash A_{n}}\right\|_{p} \rightarrow 0 \quad \text { for } n \rightarrow \infty
$$

and we can additionally assume that $A_{n}+A_{n} \subseteq A_{n+1}$. Then, for each $n$, there is an $S$-continuous function $g_{n} \in{ }^{*} \mathbb{C}^{G}$ such that $g_{n}(x)=1$ for $x \in A_{n}, g_{n}(x)=0$ for $x \in G \backslash A_{n+1}$ and $0 \leq g_{n}(x) \leq 1$ for $x \in A_{n+1} \backslash A_{n}$. We put $f_{n}=f \cdot g_{n}$. From

$$
\left\|f-f_{n}\right\|_{p} \leq\left\|f \cdot 1_{G \backslash A_{n}}\right\|_{p}
$$

it follows that $\left\|f-f_{n}\right\|_{p} \rightarrow 0$.

Let us show that $f_{n} \in \mathcal{C}_{\mathrm{c}}^{p, 1}\left(G, G_{0}, G_{\mathrm{f}}\right)$ for each $n$. Clearly, $\operatorname{supp} f_{n} \subseteq A_{n+1} \subseteq G_{\mathrm{f}}$ and $\left\|f_{n}\right\|_{p} \leq\|f\|_{p}<\infty$. According to Hölder's inequality,

$$
\left\|f_{n}\right\|_{1}=\left\|f g_{n}\right\|_{1} \leq\|f\|_{p}\left\|g_{n}\right\|_{q}<\infty
$$

where $\frac{1}{p}+\frac{1}{q}=1$. Taking any $a \in G_{0}$ we have

$$
\begin{aligned}
\left\|\left(f_{n}\right)_{a}-f_{n}\right\|_{p} & \leq\left\|f_{a}\left(\left(g_{n}\right)_{a}-g_{n}\right)\right\|_{p}+\left\|\left(f_{a}-f\right) g_{n}\right\|_{p} \\
& \leq\left\|f_{a}\right\|_{p}\left\|\left(g_{n}\right)_{a}-g\right\|_{\infty}+\left\|f_{a}-f\right\|_{p}\left\|g_{n}\right\|_{\infty} \approx 0
\end{aligned}
$$

showing that $f_{n}$ is $S^{p}$-continuous. Hence $f_{n} \in \mathcal{C}_{\mathrm{c}}^{p, 1}\left(G, G_{0}, G_{\mathrm{f}}\right)$.

Theorem 2.1.4 and Proposition 1.2.3 yield the following:

Theorem 2.1.5 Let $1 \leq p<\infty$. Then for any measurable function $\boldsymbol{f}: \mathbf{G} \rightarrow \mathbb{C}$ the following conditions are equivalent:

(i) $\boldsymbol{f} \in \mathrm{L}^{p}(\mathbf{G})$

(ii) $f$ has an $S^{p}$-integrable lifting

(iii) $f$ has an $S^{p}$-continuous lifting $f \in \mathcal{M}^{p}\left(G, G_{0}, G_{\mathrm{f}}\right)$

Remark 1 Let $1 \leq p<\infty$. We adopt a similar and equally justified convention $\mathcal{S}^{p}\left(G, G_{0}, G_{\mathrm{f}}\right)=\mathcal{S}^{p}\left(G, G_{\mathrm{f}}, d\right)$ like that for $\mathcal{M}^{p}\left(G, G_{0}, G_{\mathrm{f}}\right)$. Then, according to Theorem 2.1.4, we have

$$
\begin{aligned}
& \mathcal{L}^{p}\left(G, G_{0}, G_{\mathrm{f}}\right)=\left\{f \in \mathcal{M}^{p}\left(G, G_{0}, G_{\mathrm{f}}\right):(\forall a \in G)\left(a \approx 0 \Rightarrow\left\|f_{a}-f\right\|_{p} \approx 0\right)\right\} \\
& \mathcal{S}^{p}\left(G, G_{0}, G_{\mathrm{f}}\right)=\left\{f \in \mathcal{M}^{p}\left(G, G_{0}, G_{\mathrm{f}}\right):\left(\forall^{\text {int }} A \subseteq G\right)\left(d|A| \approx 0 \Rightarrow\left\|f \cdot 1_{A}\right\|_{p} \approx 0\right)\right\}
\end{aligned}
$$

so that the characterization of $\mathcal{L}^{p}\left(G, G_{0}, G_{\mathrm{f}}\right)$ differs from the definition of $\mathcal{S}^{p}\left(G, G_{0}, G_{\mathrm{f}}\right)$ just in replacing the condition of absolute $S$-continuity by that of $S^{p}$-continuity. As it follows from 2.1.4 and 2.1.5, $\mathcal{L}^{p}\left(G, G_{0}, G_{\mathrm{f}}\right) \subseteq \mathcal{S}^{p}\left(G, G_{0}, G_{\mathrm{f}}\right)$, ie, for a function $f \in \mathcal{M}^{p}\left(G, G_{0}, G_{\mathrm{f}}\right), S^{p}$-continuity implies absolute $S$-continuity (but not vice versa). However, one would like to have a more direct proof of this inclusion. 
Remark 2 Given any condensing IMG triplet $\left(X, E, X_{\mathrm{f}}\right)$ with hyperfinite ambient set $X$ and a nonnegative internal function $d: X \rightarrow{ }^{*} \mathbb{R}$, the observable trace $\mathbf{X}=X_{\mathrm{f}} / E$ is a Hausdorff locally compact space, so that it still makes sense to ask which internal functions $f: X \rightarrow{ }^{*} \mathbb{C}$ are liftings of functions $\boldsymbol{f} \in \mathrm{L}^{p}(\mathbf{X}, \boldsymbol{m})$, where $\boldsymbol{m}=\boldsymbol{m}_{d}$ is the Lebesgue measure on $\mathbf{X}$ obtained by pushing down the Loeb measure $\lambda_{d}$. However, as long as no group structure on $X$ is involved, $\mathcal{L}^{p}\left(X, E, X_{\mathrm{f}}\right)$ cannot be characterized in terms of $S^{p}$-continuity. It would be nice to have some reasonable intrinsic characterization of $\mathcal{L}^{p}\left(X, E, X_{\mathrm{f}}\right)$ within such a more general setting, at least for constant $d(x)=d$ such that $d|A| \not \approx 0$ for some and $d|A|<\infty$ for each internal set $A \subseteq X_{\mathrm{f}}$.

Remark 3 The characterizing conditions of $\mathcal{L}^{p}\left(G, G_{0}, G_{\mathrm{f}}\right)$ make sense also for $p=\infty$. More precisely, the conjunction of the conditions $\|f\|_{\infty}<\infty,\left\|f \cdot 1_{Z}\right\|_{\infty} \approx 0$ for each internal set $Z \subseteq G \backslash G_{\mathrm{f}}$ and $S^{\infty}$-continuity defines the subspace $\mathcal{C}_{0}\left(G, G_{0}, G_{\mathrm{f}}\right)$ of $S$-continuous internal functions $f: G \rightarrow{ }^{*} \mathbb{C}$ which are finite on the whole $G$ and infinitesimal outside of $G_{\mathrm{f}}$. Thus we could formally write $\mathcal{L}^{\infty}\left(G, G_{0}, G_{\mathrm{f}}\right)=$ $\mathcal{C}_{0}\left(G, G_{0}, G_{\mathrm{f}}\right)$. This, however, would interfere with the adopted standard notation, as such an $\mathcal{L}^{\infty}\left(G, G_{0}, G_{\mathrm{f}}\right)$ would be formed just by the liftings of functions in $\mathrm{C}_{0}(\mathbf{G})$ which is a proper closed subspace of the Banach space $\mathrm{L}^{\infty}(\mathbf{G})$ (cf Proposition 1.1.1).

\subsection{The Smoothness-and-Decay Principle}

The more smooth is a function $f: \mathbb{R}^{n} \rightarrow \mathbb{C}$, the more rapidly its Fourier transform $\widehat{f}: \mathbb{R}^{n} \rightarrow \mathbb{C}$ decays; conversely, the more rapidly a function $f: \mathbb{R}^{n} \rightarrow \mathbb{C}$ decays, the smoother is its Fourier transform $\widehat{f}: \mathbb{R}^{n} \rightarrow \mathbb{C}$. This vague informal statement is known as the Smoothness-and-Decay Principle and-jointly with the Uncertainty Principle to which it is closely related-it belongs to fundamental heuristic principles of the Fourier or time-frequency analysis. It can take the form of various precise mathematical statements, some of which generalize from $\mathbb{R}$ or $\mathbb{R}^{n}$ to arbitrary LCA groups (see, eg, Gröchenig [8] and Tao [17] for discussion).

The view through the lens of an IMG group triplet $\left(G, G_{0}, G_{\mathrm{f}}\right)$ with hyperfinite abelian ambient group $G$ and its dual triplet $\left(\widehat{G}, G_{\mathrm{f}}^{\downarrow}, G_{0}^{\downarrow}\right)$ offers an intuitively appealing explanation of this principle for internal functions $f: G \rightarrow{ }^{*} \mathbb{C}$, based on the Fourier inversion formula

$$
f(x)=\widehat{d} \sum_{\gamma \in \widehat{G}} \widehat{f}(\gamma) \gamma(x)
$$

in which both $S$-continuous characters $\gamma \in G_{0}^{\downarrow}$ as well as non- $S$-continuous characters $\gamma \in \widehat{G} \backslash G_{0}^{\downarrow}$ occur. If $f$ is smooth or continuous (in some intuitive meaning of these 
words) then the contribution of the non- $S$-continuous characters to the above expansion of $f$ must be negligible in some sense. This condition causes a kind of quick decay of $\widehat{f}$. The other way around, viewing the elements $x \in G$ as characters of the dual group $\widehat{G}$, the Fourier transform of $f$ can be expressed as their linear combination:

$$
\widehat{f}(\gamma)=d \sum_{x \in G} f(x) \bar{\gamma}(x)=d \sum_{x \in G} f(-x) x(\gamma)
$$

If $f$ decays quickly, ie if the values of $f$ on the infinite elements $x \in G \backslash G_{\mathrm{f}}$ are somehow negligible, then the values of its Fourier transform are essentially determined by the values of $f$ on the finite elements $x \in G_{\mathrm{f}}$ which happen to coincide with the $S$-continuous characters of $\widehat{G}$ by the Triplet Duality Theorem-see Zlatoš [19, Theorem 2.2.5]. If additionally none of the coefficients $f(x)$ for $x \in G_{\mathrm{f}}$ are too big then we can reasonably expect $\widehat{f}$ to be smooth or continuous in some sense.

The next theorem is a fairly general precise statement of this form of the Smoothnessand-Decay Principle. Both its formulation as well as its proof borrow some ideas from a paper by Pego [14].

A pair of internal norms $\mathrm{N}$ on $* \mathbb{C}^{G}$ and $\mathrm{M}$ on $* \mathbb{C}^{\widehat{G}}$ is called Fourier compatible if the Fourier transform $\mathcal{F}:{ }^{*} \mathbb{C}^{G} \rightarrow{ }^{*} \mathbb{C} \widehat{G}$ is a bounded linear operator with respect to the norms $\mathrm{N}, \mathrm{M}$, ie,

$$
\mathrm{N}(f)<\infty \Rightarrow \mathrm{M}(\widehat{f})<\infty
$$

for each $f \in{ }^{*} \mathbb{C}^{G}$. This is equivalent to the $S$-continuity of $\mathcal{F}$, ie:

$$
\mathrm{N}(f) \approx 0 \Rightarrow \mathrm{M}(\widehat{f}) \approx 0
$$

Recall from [19, Section 2.1] that a norm $\mathrm{N}$ on ${ }^{*} \mathbb{C}^{G}$ is called absolute if $\mathrm{N}(f) \leq \mathrm{N}(g)$ for any $f, g \in *^{*} \mathbb{C}^{G}$ such that $|f(x)| \leq|g(x)|$ for all $x \in G$.

Theorem 2.2.1 (The Smoothness-and-Decay Principle) Let N, M be Fourier compatible internal norms on the linear spaces $* \mathbb{C}^{G},{ }^{*} \mathbb{C}^{\widehat{G}}$, respectively. Then for every function $f \in{ }^{*} \mathbb{C}^{G}$ the following implications hold:

(i) If $\mathrm{M}$ is absolute and $f$ is $S^{\mathrm{N}}$-continuous then $\mathrm{M}\left(\widehat{f} \cdot 1_{\Gamma}\right) \approx 0$ for every internal set $\Gamma \subseteq \widehat{G} \backslash G_{0}^{\downarrow}$.

(ii) If $\mathrm{N}$ is absolute, $\mathrm{N}(f)<\infty$ and $\mathrm{N}\left(f \cdot 1_{X}\right) \approx 0$ for every internal set $X \subseteq G \backslash G_{\mathrm{f}}$ then $\widehat{f}$ is $S^{\mathrm{M}}$-continuous.

Proof (a) In this part of proof we will once more make use of the families of internal functions $h_{\varrho r}$ and $\vartheta_{\varrho r}$ (see Lemma 2.1.2 and the text immediately preceding it). 
Assume that $\mathrm{N}\left(f_{a}-f\right) \approx 0$ for any $a \in G_{0}$. We will show that $\mathrm{M}\left(\widehat{f} \cdot 1_{\Gamma}\right) \approx 0$ for every internal set $\Gamma \subseteq \widehat{G} \backslash G_{0}^{\downarrow}$. Let us fix any (standard) $t \in(0,1)$. By [19, Corollary 2.3.2], $\Gamma \cap \operatorname{Spec}_{t}\left(h_{\varrho}\right)=\emptyset$ for every $\varrho \in \mathcal{V}$ and standard $r>0$. As $\mathcal{V}$ is upward directed, by saturation there are $\tau \in{ }^{*} \mathcal{V}$, satisfying $\varrho \leq \tau$ for all $\varrho \in \mathcal{V}$, and a positive $s \approx 0$, such that $\Gamma \cap \operatorname{Spec}_{t}\left(h_{\tau s}\right)=\emptyset$ still holds. Let us denote $\Delta=\widehat{G} \backslash \operatorname{Spec}_{t}\left(h_{\tau s}\right)$ and recall that $\vartheta_{\tau s}=\left\|h_{\tau s}\right\|_{1}^{-1} h_{\tau s}$. As $h_{\tau s}$ is even and nonnegative, so is $\vartheta_{\tau s}$, hence

$$
\widehat{\vartheta}_{\tau s}\left(1_{G}\right)=d \sum_{a \in G} \vartheta_{\tau s}(a)=\left\|\vartheta_{\tau s}\right\|_{1}=1
$$

and for $\gamma \in \Delta$ we have

hence

$$
\begin{gathered}
\left|\widehat{\vartheta}_{\tau s}(\gamma)\right|=|| h_{\tau s} \|_{1}^{-1}\left|\widehat{h}_{\tau s}(\gamma)\right|<t \\
1-t<1-\left|\widehat{\vartheta}_{\tau s}(\gamma)\right| \leq\left|1-\widehat{\vartheta}_{\tau s}(\gamma)\right| .
\end{gathered}
$$

Since $\Gamma \subseteq \Delta$ and the norm $\mathrm{M}$ is absolute:

$$
(1-t) \mathrm{M}\left(\widehat{f} \cdot 1_{\Gamma}\right) \leq(1-t) \mathrm{M}\left(\widehat{f} \cdot 1_{\Delta}\right) \leq \mathrm{M}\left(\left(1_{\widehat{G}}-\widehat{\vartheta}_{\tau s}\right) \widehat{f}\right)=\mathrm{M}\left(\left(f-\vartheta_{\tau s} * f\right)^{\uparrow}\right)
$$

Due to our choice of $\tau$ and $s$ we have $B_{\tau}(s) \subseteq G_{0}$; consequently, $\mathrm{N}\left(f-\vartheta_{\tau s} * f\right) \approx 0$ by Lemma 2.1.2. As the norms $\mathrm{N}, \mathrm{M}$ are Fourier compatible, this implies that $\mathrm{M}\left(\left(f-\vartheta_{\tau s} * f\right)^{\wedge}\right) \approx 0$, as well. Since $t \not \approx 1$, we can conclude that $\mathrm{M}\left(\widehat{f} \cdot 1_{\Gamma}\right) \approx 0$.

(b) Assume that $\mathrm{N}(f)$ is finite and $\mathrm{N}\left(f \cdot 1_{X}\right) \approx 0$ for each internal set $X \subseteq G \backslash G_{\mathrm{f}}$. We are to show that $\mathrm{M}\left(\widehat{f}_{\gamma}-\widehat{f}\right) \approx 0$ for any $\gamma \in G_{\mathrm{f}}^{\downarrow}$. First notice that:

$$
\widehat{f}_{\gamma}-\widehat{f}=\left(\left(\gamma-1_{G}\right) f\right)^{\widehat{ }}
$$

As $\gamma \in G_{\mathrm{f}}^{\downarrow}, \gamma(x) \approx 1$ for each $x \in G_{\mathrm{f}}$. Due to saturation, there is an internal set $Y$ such that $G_{\mathrm{f}} \subseteq Y \subseteq G$ and $\gamma(y) \approx 1$ for each $y \in Y$; then $X=G \backslash Y \subseteq G \backslash G_{\mathrm{f}}$. Let us denote:

$$
\varepsilon=\left\|\left(\gamma-1_{G}\right) \cdot 1_{Y}\right\|_{\infty}=\max _{y \in Y}|\gamma(y)-1|
$$

Obviously, $\varepsilon \approx 0$. As $\mathrm{N}$ is absolute:

$$
\begin{aligned}
\mathrm{N}\left(\left(\gamma-1_{G}\right) f\right) & \leq \mathrm{N}\left(\left(\gamma-1_{G}\right) f \cdot 1_{Y}\right)+\mathrm{N}\left(\left(\gamma-1_{G}\right) f \cdot 1_{X}\right) \\
& \leq \varepsilon \mathrm{N}(f)+2 \mathrm{~N}\left(f \cdot 1_{X}\right) \approx 0
\end{aligned}
$$

Therefore, $\mathrm{M}\left(\widehat{f}_{\gamma}-\widehat{f}\right) \approx 0$ as well.

The last theorem applies to any pair of norms $\|\cdot\|_{p}$ on $* \mathbb{C}^{G}$ and $\|\cdot\|_{q}$ on $* \mathbb{C}^{\widehat{G}}$ for $1 \leq p \leq 2$ and $q=p /(p-1)$, including $p=1, q=\infty$, in which case we have: 
Corollary 2.2.2 For every function $f \in{ }^{*} \mathbb{C}^{G}$ the following conditions hold:

(a) If $f$ is $S^{1}$-continuous then $\widehat{f}(\gamma) \approx 0$ for all $\gamma \in \widehat{G} \backslash G_{0}^{\downarrow}$

(b) If $\|f\|_{1}<\infty$ and $\left\|f \cdot 1_{X}\right\|_{1} \approx 0$ for every internal set $X \subseteq G \backslash G_{\mathrm{f}}$ then $\widehat{f}$ is S-continuous, ie, $\widehat{f}(\gamma) \approx \widehat{f}(\chi)$ for all $\gamma, \chi \in \widehat{G}$ such that $\gamma(x) \approx \chi(x)$ for each $x \in G_{\mathrm{f}}$

(c) $\mathcal{F}\left[\mathcal{L}^{1}\left(G, G_{0}, G_{\mathrm{f}}\right)\right] \subseteq \mathcal{C}_{0}\left(\widehat{G}, G_{\mathrm{f}}^{\downarrow}, G_{0}^{\downarrow}\right)$

Notice that (c) is a hyperfinite dimensional version of the Riemann-Lebesgue lemma and (b) can be written as a similar inclusion:

$$
\mathcal{F}\left[\mathcal{M}\left(G, G_{0}, G_{\mathrm{f}}\right)\right] \subseteq \mathcal{C}_{\text {bu }}\left(\widehat{G}, G_{\mathrm{f}}^{\downarrow}\right)
$$

Corollary 2.2.3 Let $1<p \leq 2$ and $q=p /(p-1)$ be its dual exponent. Then for every function $f \in{ }^{*} \mathbb{C}^{G}$ the following conditions hold:

(a) If $f$ is $S^{p}$-continuous then $\left\|\widehat{f} \cdot 1_{\Gamma}\right\|_{q} \approx 0$ for every internal set $\Gamma \subseteq \widehat{G} \backslash G_{0}^{\downarrow}$

(b) If $\|f\|_{p}<\infty$ and $\left\|f \cdot 1_{X}\right\|_{p} \approx 0$ for every internal set $X \subseteq G \backslash G_{\mathrm{f}}$ then $\widehat{f}$ is $S^{q}$-continuous, ie $\left\|\widehat{f}_{\gamma}-\widehat{f}\right\|_{q} \approx 0$ for all $\gamma \in G_{\mathrm{f}}^{\downarrow}$

(c) $\mathcal{F}\left[\mathcal{L}^{p}\left(G, G_{0}, G_{\mathrm{f}}\right)\right] \subseteq \mathcal{L}^{q}\left(\widehat{G}, G_{\mathrm{f}}^{\downarrow}, G_{0}^{\downarrow}\right)$

In the Hilbert space case $p=q=2$ the last corollary can be slightly strengthened. Applying 2.2.3 both to the Fourier transform $\mathcal{F}:{ }^{*} \mathbb{C}^{G} \rightarrow{ }^{*} \mathbb{C}^{\widehat{G}}$ and its inverse $\mathcal{F}^{-1}:{ }^{*} \mathbb{C}^{\widehat{G}} \rightarrow{ }^{*} \mathbb{C}^{G}$ for functions satisfying $\|f\|_{2}<\infty$, we get equivalences in (a), (b) and equality in (c).

Corollary 2.2.4 For every function $f \in{ }^{*} \mathbb{C}^{G}$ such that $\|f\|_{2}<\infty$ the following conditions hold:

(a) $f$ is $S^{2}$-continuous if and only if $\left\|\widehat{f} \cdot 1_{\Gamma}\right\|_{2} \approx 0$ for every internal set $\Gamma \subseteq \widehat{G} \backslash G_{0}^{\downarrow}$

(b) $\left\|f \cdot 1_{X}\right\|_{2} \approx 0$ for every internal set $X \subseteq G \backslash G_{\mathrm{f}}$ if and only if $\widehat{f}$ is $S^{2}$-continuous

(c) $\mathcal{F}\left[\mathcal{L}^{2}\left(G, G_{0}, G_{\mathrm{f}}\right)\right]=\mathcal{L}^{2}\left(\widehat{G}, G_{\mathrm{f}}^{\downarrow}, G_{0}^{\downarrow}\right)$

Note that the smoothness and decay conditions in the last three corollaries can be viewed as nonstandard (infinitesimal) analogues of the Kolmogoroff-Riesz-Tamarkin criteria for relative compactness in the Lebesgue spaces $L^{p}(\mathbf{G})$ (see Dinculeanu [4], Pego [14]).

The following result follows directly from Corollary 2.2.4. 
Corollary 2.2.5 For every function $f \in{ }^{*} \mathbb{C}^{G}$ such that $\|f\|_{2}<\infty$ the following conditions are equivalent:

(i) $f \in \mathcal{L}^{2}\left(G, G_{0}, G_{\mathrm{f}}\right)$

(ii) $\widehat{f} \in \mathcal{L}^{2}\left(\widehat{G}, G_{\mathrm{f}}^{\perp}, G_{0}^{\downarrow}\right)$

(iii) Both $f$ and $\widehat{f}$ are $S^{2}$-continuous

(iv) $\left\|f \cdot 1_{X}\right\|_{2} \approx 0$ for every internal set $X \subseteq G \backslash G_{\mathrm{f}}$ and $\left\|\widehat{f} \cdot 1_{\Gamma}\right\|_{2} \approx 0$ for every internal set $\Gamma \subseteq \widehat{G} \backslash G_{0}^{\downarrow}$

Corollary 2.2.5 generalizes a result by Albeverio, Gordon and Khrennikov [2], where the equivalence of conditions (i), (ii) and (iv) in case there is an internal subgroup $K$ of $G$ such that $G_{0} \subseteq K \subseteq G_{\mathrm{f}}$ was proved. This assumption is equivalent to the existence of a compact open subgroup of $G^{b}$. It is also mentioned there without proof that the group of reals $\mathbb{R}$ can be represented as well as $\mathbb{R} \cong G^{b}=G_{\mathrm{f}} / G_{0}$ for some triplet $\left(G, G_{0}, G_{\mathrm{f}}\right)$ satisfying their restricted version of Corollary 2.2.5.

\subsection{Generalized Gordon's Conjecture 3: hyperfinite dimensional approxi- mation of Fourier transforms}

Various versions of the Smoothness-and-Decay Principle proved in the previous section make it possible to approximate the classical Fourier transforms on various function spaces related to the LCA group $\mathbf{G}=G_{\mathrm{f}} / G_{0}$ by the discrete Fourier transform on the hyperfinite dimensional linear space ${ }^{*} \mathbb{C}^{G}$. We denote by $\mathbf{F}(\boldsymbol{f})=\widehat{\boldsymbol{f}}$ the classical Fourier transform of a function $\boldsymbol{f}: \mathbf{G} \rightarrow \mathbb{C}$ and by $\mathcal{F}(f)=\widehat{f}$ the discrete Fourier transform of an internal function $f: G \rightarrow{ }^{*} \mathbb{C}$.

The discrete hyperfinite dimensional Fourier transform $\mathcal{F}:{ }^{*} \mathbb{C}^{G} \rightarrow{ }^{*} \mathbb{C}^{\widehat{G}}$ approximates the classical Fourier transform $\mathbf{F}: \mathrm{L}^{1}(\mathbf{G}) \rightarrow \mathrm{C}_{0}(\widehat{\mathbf{G}})$ in the following sense:

Theorem 2.3.1 (The HFD Fourier Transform Approximation Theorem) Let the internal function $f \in \mathcal{L}^{1}\left(G, G_{0}, G_{\mathrm{f}}\right)$ be a lifting of a function $f \in \mathrm{L}^{1}(\mathbf{G})$. Then the internal function $\mathcal{F}(f)=\widehat{f} \in \mathcal{C}_{0}\left(\widehat{G}, G_{\mathrm{f}}^{\downarrow}, G_{0}^{\downarrow}\right)$ is a lifting of the function $\mathbf{F}(\boldsymbol{f})=\widehat{\boldsymbol{f}} \in$ $\mathrm{C}_{0}(\widehat{\mathbf{G}})$.

Proof Let $f \in \mathcal{L}^{1}\left(G, G_{0}, G_{\mathrm{f}}\right)$ be a lifting of $\boldsymbol{f} \in \mathrm{L}^{1}(\mathbf{G})$. Then $\widehat{f} \in \mathcal{C}_{0}\left(\widehat{G}, G_{\mathrm{f}}^{\downarrow}, G_{0}^{\downarrow}\right)$ by Corollary 2.2.2(c). Thus it suffices to prove that

$$
\widehat{\boldsymbol{f}}\left(\gamma^{b}\right)=\circ \widehat{f}(\gamma)
$$


for each $\gamma \in G_{0}^{\downarrow}$. However, as $\gamma$ is bounded and $S$-continuous, ie, $\gamma \in \mathcal{C}_{\text {bu }}\left(G, G_{0}\right)$, it is routine to check that the internal function $f \bar{\gamma} \in \mathcal{L}^{1}\left(G, G_{0}, G_{\mathrm{f}}\right)$ is a lifting of the function $\boldsymbol{f} \bar{\gamma}^{b} \in \mathrm{L}^{1}(\widehat{\mathbf{G}})$. Then by Ziman and Zlatoš [18, Proposition 3.5]:

$$
\widehat{\boldsymbol{f}}\left(\gamma^{b}\right)=\int \boldsymbol{f} \bar{\gamma}^{b} \mathrm{~d} \boldsymbol{m}={ }^{\circ}\left(d \sum_{x \in G} f(x) \bar{\gamma}(x)\right)={ }^{\circ} \widehat{f}(\gamma)
$$

See also the text preceding Proposition 1.2.3.)

For $1<p \leq 2$ and $1 / p+1 / q=1$ the Fourier transform $\mathbf{F}: \mathrm{L}^{p}(\mathbf{G}) \rightarrow \mathrm{L}^{q}(\widehat{\mathbf{G}})$ is defined as the continuous extension (with respect to the norms $\|\cdot\|_{p}$ on $\mathrm{L}^{p}(\mathbf{G})$ and $\|\cdot\|_{q}$ on $\left.\mathrm{L}^{q}(\widehat{\mathbf{G}})\right)$ of the restriction of the Fourier transform $\mathbf{F}: \mathrm{L}^{1}(\mathbf{G}) \rightarrow \mathrm{C}_{0}(\widehat{\mathbf{G}})$ to the dense subspace $\mathrm{L}^{p}(\mathbf{G}) \cap \mathrm{L}^{1}(\mathbf{G})$ of $\mathrm{L}^{p}(\mathbf{G})$ (see Hewitt and Ross [11], Loomis [12] or Rudin [16]). For functions in this subspace everything works as in the proof above. Thus, by a continuity argument Theorem 2.3.1 together with Corollary 2.2.3(c) give rise to HFD approximations of the classical Fourier transforms $\mathbf{F}: \mathrm{L}^{p}(\mathbf{G}) \rightarrow \mathrm{L}^{q}(\widehat{\mathbf{G}})$ in a similar way. The case $p=q=2$ of the Fourier-Plancherel transform $\mathbf{F}: \mathrm{L}^{2}(\mathbf{G}) \rightarrow \mathrm{L}^{2}(\widehat{\mathbf{G}})$ settles Gordon's Conjecture 3.

Theorem 2.3.2 (The Generalized Gordon Conjecture 3) Let $p \in(1,2]$ and $q \in[2, \infty)$ be its dual exponent. Let the internal function $f \in \mathcal{L}^{p}\left(G, G_{0}, G_{\mathrm{f}}\right)$ be a lifting of a function $\boldsymbol{f} \in \mathrm{L}^{p}(\mathbf{G})$. Then the internal function $\mathcal{F}(f)=\widehat{f} \in \mathcal{L}^{q}\left(\widehat{G}, G_{\mathrm{f}}^{\downarrow}, G_{0}^{\downarrow}\right)$ is a lifting of the function $\mathbf{F}(\boldsymbol{f})=\widehat{\boldsymbol{f}} \in \mathrm{L}^{q}(\widehat{\mathbf{G}})$.

The HFD Fourier Transform Approximation Theorem 2.3.1 extends to the FourierStieltjes transform $\mathbf{F}: \mathrm{M}(\mathbf{G}) \rightarrow \mathrm{C}_{\mathrm{bu}}(\widehat{\mathbf{G}})$, as well.

Theorem 2.3.3 (The HFD Fourier-Stieltjes Transform Approximation Theorem) Let the internal function $g \in \mathcal{M}\left(G, G_{0}, G_{\mathrm{f}}\right)$ be a weak lifting of a complex regular Borel measure $\boldsymbol{\mu} \in \mathrm{M}(\mathbf{G})$. Then the internal function $\mathcal{F}(g)=\widehat{g} \in \mathcal{C}_{\mathrm{bu}}\left(\widehat{G}, G_{\mathrm{f}}^{\downarrow}\right)$ is a lifting of the function $\mathbf{F}(\boldsymbol{\mu})=\widehat{\boldsymbol{\mu}} \in \mathrm{C}_{\mathrm{bu}}(\widehat{\mathbf{G}})$.

Proof Let $g \in \mathcal{M}\left(G, G_{0}, G_{\mathrm{f}}\right)$ be a weak lifting of $\boldsymbol{\mu} \in \mathrm{M}(\mathbf{G})$. Then $\widehat{g} \in \mathcal{C}_{\mathrm{bu}}\left(\widehat{G}, G_{\mathrm{f}}^{\downarrow}\right)$ by Corollary 2.2.2(b). Thus it suffices to prove that

$$
\widehat{\boldsymbol{\mu}}\left(\gamma^{b}\right)={ }^{\circ} \widehat{g}(\gamma)
$$

for each $\gamma \in G_{0}^{\downarrow}$. For the same reason as in the proof of Theorem 2.3.1 we have

$$
\int f^{b} \mathrm{~d} \boldsymbol{\mu}={ }^{\circ}\left(d \sum_{x \in G} f(x) g(x)\right)
$$


for every internal function $f \in \mathcal{C}_{\mathrm{b}}\left(G, G_{0}, G_{\mathrm{f}}\right)$. For $f=\bar{\gamma} \in G_{0}^{\downarrow} \subseteq \mathcal{C}_{\mathrm{bu}}\left(G, G_{0}\right)$ this gives

$$
\widehat{\boldsymbol{\mu}}\left(\gamma^{b}\right)=\int \bar{\gamma}^{b} \mathrm{~d} \boldsymbol{\mu}={ }^{\circ}\left(d \sum_{x \in G} \bar{\gamma}(x) g(x)\right)={ }^{\circ} \widehat{g}(\gamma)
$$

In particular, if $\boldsymbol{g} \in \mathrm{L}^{1}(\mathbf{G}), \mathrm{d} \boldsymbol{\mu}=\boldsymbol{g} \mathrm{d} \boldsymbol{m}$ and $g \in \mathcal{L}^{1}\left(G, G_{0}, G_{\mathrm{f}}\right)$ is a lifting of $\boldsymbol{g}$ then

$$
\widehat{\boldsymbol{g}}\left(\gamma^{b}\right)=\widehat{\boldsymbol{\mu}}\left(\gamma^{b}\right)=\int \bar{\gamma}^{b} \boldsymbol{g} \mathrm{d} \boldsymbol{m}={ }^{\circ}\left(d \sum_{x \in G} \bar{\gamma}(x) g(x)\right)={ }^{\circ} \widehat{g}(\gamma)
$$

for each $\gamma \in G_{0}^{\downarrow}$, reproving Theorem 2.3.1. This account indicates that it is Theorem 2.3.3 which is crucial for hyperfinite dimensional approximations of the Fourier transform on LCA groups. Therefore we address the issue raised in the remark closing the introductory part of Zlatoš [19, Section 2.5] primarily for the Fourier-Stieltjes transform.

Assume, for the rest of this section, that $\left(G, G_{0}, G_{\mathrm{f}}\right)$ is an IMG group triplet with hyperfinite abelian ambient group $G$, arising from an HFI approximation $\eta: G \rightarrow{ }^{*} \mathbf{G}$ of the Hausdorff LCA group G. Let us denote $\mathcal{F}_{\eta}:{ }^{*} \mathbb{C}^{G} \rightarrow{ }^{*} \mathbb{C}^{*} \widehat{\mathbf{G}}$ the internal linear operator given by

$$
\mathcal{F}_{\eta}(f)(\chi)=\langle f, \chi \circ \eta\rangle=d \sum_{x \in G} f(x) \bar{\chi}(\eta x)
$$

for $f \in{ }^{*} \mathbb{C}^{G}, \chi \in{ }^{*} \widehat{\mathbf{G}}$. The modified discrete Fourier transform $\mathcal{F}_{\eta}$, defined by means of the internal inner product on ${ }^{*} \mathbb{C}^{G}$, can be employed for the approximation of the classical Fourier transform on $\mathbf{G}$ without the need to mention the adjoint HFI approximation $\phi: \widehat{G} \rightarrow{ }^{*} \widehat{\mathbf{G}}$ of the dual group $\widehat{G}$.

Proposition 2.3.4 Let $\mathbf{F}: \mathrm{M}(\mathbf{G}) \rightarrow \mathrm{C}_{\mathrm{bu}}(\widehat{\mathbf{G}})$ be the Fourier-Stieltjes transform on $\mathbf{G}$, $\boldsymbol{\mu} \in \mathbf{M}(\mathbf{G})$ and $g \in \mathcal{M}\left(G, G_{0}, G_{\mathrm{f}}\right)$ be a weak lifting of $\boldsymbol{\mu}$. Then

$$
\mathbf{F}(\boldsymbol{\mu})(\gamma)=\widehat{\boldsymbol{\mu}}(\gamma) \approx \mathcal{F}_{\eta}(g)\left({ }^{*} \gamma\right)
$$

for each $\gamma \in \widehat{\mathbf{G}}$.

Proof As ${ }^{*} \gamma \circ \eta$ is almost homomorphic and $S$-continuous on $G_{\mathrm{f}}$, by [19, Theorem 2.2.4] there is $\gamma \in G_{0}^{\downarrow}$ such that ${ }^{*} \gamma(\eta x) \approx \gamma(x)$ for each $x \in G_{\mathrm{f}}$. According to Theorem 2.3.3:

$$
\widehat{\boldsymbol{\mu}}(\gamma) \approx \widehat{g}(\gamma)=\langle g, \gamma\rangle
$$


Due to saturation there is an internal set $X$ such that $G_{\mathrm{f}} \subseteq X \subseteq G$ and ${ }^{*} \gamma(\eta x) \approx \gamma(x)$ holds for all $x \in X$. Denoting $Y=G \backslash X$ we have

$$
\begin{aligned}
\left|\left\langle g,{ }^{*} \gamma \circ \eta\right\rangle-\langle g, \gamma\rangle\right| & \leq\left|\left\langle g,\left({ }^{*} \gamma \circ \eta-\gamma\right) \cdot 1_{X}\right\rangle\right|+\left|\left\langle g \cdot 1_{Y},{ }^{*} \gamma \circ \eta-\gamma\right\rangle\right| \\
& \leq\|g\|\left\|_{1}\right\|\left({ }^{*} \gamma \circ \eta-\gamma\right) \cdot 1_{X}\left\|_{\infty}+\right\| g \cdot 1_{Y}\left\|_{1}\right\|^{*} \gamma \circ \eta-\gamma \|_{\infty} \approx 0
\end{aligned}
$$

as both $\|g\|_{1}$ and $\left\|^{*} \gamma \circ \eta-\gamma\right\|_{\infty}$ are finite, and both $\left\|\left({ }^{*} \gamma \circ \eta-\gamma\right) \cdot 1_{X}\right\|_{\infty}$ and $\left\|g \cdot 1_{Y}\right\|_{1}$ are infinitesimal. The conclusion $\mathcal{F}_{\eta}(g)\left({ }^{*} \gamma\right)=\left\langle g,{ }^{*} \boldsymbol{\gamma} \circ \eta\right\rangle \approx \widehat{\boldsymbol{\mu}}(\boldsymbol{\gamma})$ is now obvious.

Proposition 2.3.4, jointly with Theorems 2.3.1 and 2.3.2, respectively, yield the following two corollaries.

Corollary 2.3.5 Let $\mathbf{F}: \quad \mathrm{L}^{1}(\mathbf{G}) \rightarrow \mathrm{C}_{0}(\widehat{\mathbf{G}})$ be the Fourier transform on $\mathbf{G}, \boldsymbol{f} \in \mathrm{L}^{1}(\mathbf{G})$ and $f \in \mathcal{L}^{1}\left(G, G_{0}, G_{\mathrm{f}}\right)$ be a lifting of $f$. Then

$$
\mathbf{F}(\boldsymbol{f})(\gamma)=\widehat{\boldsymbol{f}}(\gamma) \approx \mathcal{F}_{\eta}(f)\left({ }^{*} \gamma\right)
$$

for each $\gamma \in \widehat{\mathbf{G}}$.

Corollary 2.3.6 Let $1<p \leq 2 \leq q<\infty$ be dual exponents and $\mathbf{F}: \mathrm{L}^{p}(\mathbf{G}) \rightarrow \mathrm{L}^{q}(\widehat{\mathbf{G}})$ be the Fourier transform on $\mathbf{G}$. Let further $\boldsymbol{f} \in \mathrm{L}^{p}(\mathbf{G})$ and $f \in \mathcal{L}^{p}\left(G, G_{0}, G_{\mathrm{f}}\right)$ be a lifting of $\boldsymbol{f}$. Then

$$
\mathbf{F}(\boldsymbol{f})(\gamma) \approx \mathcal{F}_{\eta}(f)\left({ }^{*} \gamma\right)
$$

for almost all $\gamma \in \widehat{\mathbf{G}}$ with respect to the Haar measure on $\widehat{\mathbf{G}}$.

\subsection{Some standard analogues}

The reader might naturally expect that we will derive some standard analogues of the nonstandard hyperfinite dimensional Fourier approximation theorems from Section 2.3. Then, as usual in such cases, these standard results would be "highly existential" and giving no explicit bounds for the precision of the approximations. Therefore, it is surprising that, given a Hausdorff LCA group $\mathbf{G}$ and a function $\boldsymbol{f} \in \mathrm{L}^{1}(\mathbf{G})$ or a measure $\boldsymbol{\mu} \in \mathrm{M}(\mathbf{G})$, we can explicitly describe some functions $f, g: G \rightarrow \mathbb{C}$ defined on some finite abelian group $G$ approximating $\boldsymbol{f}$ or $\boldsymbol{\mu}$, such that their discrete Fourier transforms $\widehat{f}=\mathcal{F}(f), \widehat{g}=\mathcal{F}(g)$ approximate the Fourier transforms $\widehat{\boldsymbol{f}}=\mathbf{F}(\boldsymbol{f}), \widehat{\boldsymbol{\mu}}=\mathbf{F}(\boldsymbol{\mu})$, respectively. Moreover, we are able to give some explicit bounds for the precision of the approximations of the Fourier transforms $\mathbf{F}(\boldsymbol{f})$ or $\mathbf{F}(\boldsymbol{\mu})$ in terms of the norm $\|\boldsymbol{f}\|_{1}$ or the total variation $\|\boldsymbol{\mu}\|$ and a parameter $\varepsilon>0$, given in advance, describing the precision of the approximation of $\boldsymbol{f}$ or $\boldsymbol{\mu}$, respectively, by $f$ or $g$. These results 
(Theorems 2.4.4 and 2.4.5) depend just on the Adjoint Approximation Scheme from Zlatoš [19, Theorem 2.5.5], so that the only nonstandard ingredient they are based on is the Adjoint Hyperfinite LCA Group Approximation Theorem [19, Corollary 2.5.2] from which Theorem 2.5.5 follows. For a compactly supported continuous function $\boldsymbol{f}: \mathbf{G} \rightarrow \mathbb{C}$ (or, more generally, for $\boldsymbol{f} \in \mathrm{C}_{0}(\mathbf{G}) \cap \mathrm{L}^{1}(\mathbf{G})$ or $\boldsymbol{f} \in \mathrm{C}_{0}(\mathbf{G}) \cap \mathrm{L}^{p}(\mathbf{G})$ ) even "nicer" discrete approximations are available. The corresponding estimates (Theorems 2.4.7 and 2.4.8), however, rest on an additional nonstandard result, namely on the fact that the Haar measure on $\mathbf{G}$ can be obtained by pushing down the Loeb on an hyperfinite approximating group $G$ constructed from a properly normalized counting measure on $G$.

Taking advantage of its generality, we will start with the approximation of the FourierStieltjes transform $\mathbf{F}: \mathbf{M}(\mathbf{G}) \rightarrow \mathrm{C}_{\text {bu }}(\widehat{\mathbf{G}})$.

Assume that $\mathbf{X}$ is a Hausdorff locally compact space whose topology is induced by some uniformity $\mathcal{U},(\mathbf{K}, \mathbf{U})$ is an $\mathbf{X}$-raster and $\eta: X \rightarrow \mathbf{X}$ is a finite $(\mathbf{K}, \mathbf{U})$ approximation of $\mathbf{X}$. An $\eta$-tagged $\mathbf{U}$-fine Borel partition of $\mathbf{K}$, briefly a tagged Borel $(\mathbf{U}, \eta)$ partition of $\mathbf{K}$, is a finite family $\boldsymbol{\pi}=\left\{\left(\mathbf{P}_{1}, x_{1}\right), \ldots,\left(\mathbf{P}_{n}, x_{n}\right)\right\}$ where $\mathbf{P}_{i}$ are nonempty pairwise disjoint Borel sets such that

$$
\mathbf{K}=\mathbf{P}_{1} \cup \ldots \cup \mathbf{P}_{n}
$$

and the elements $x_{i} \in X$ satisfy $\mathbf{P}_{i} \subseteq \mathbf{U}\left[\eta\left(x_{i}\right)\right]$ as well as $\eta(x)=\eta\left(x_{i}\right)$ for all $i \leq n$ and $x \in \eta^{-1}\left[\mathbf{P}_{i}\right]$. (The reader should notice that $\eta\left(x_{i}\right) \notin \mathbf{P}_{i}$, in which case $\eta^{-1}\left[\mathbf{P}_{i}\right]=\emptyset$, may still happen.)

Lemma 2.4.1 For every $\mathbf{X}$-raster $(\mathbf{K}, \mathbf{U})$ there exist a finite $(\mathbf{K}, \mathbf{U})$ approximation $\eta: X \rightarrow \mathbf{X}$ of $\mathbf{X}$ and an $\eta$-tagged $\mathbf{U}$-fine Borel partition $\boldsymbol{\pi}=\left\{\left(\mathbf{P}_{1}, x_{1}\right), \ldots,\left(\mathbf{P}_{n}, x_{n}\right)\right\}$ of $\mathbf{K}$.

Proof There exists an entourage $\mathbf{V} \subseteq \mathbf{U}$ which is an open subset of $\mathbf{X} \times \mathbf{X}$. Let $\eta: X \rightarrow \mathbf{X}$ be a finite $(\mathbf{K}, \mathbf{V})$ approximation of $\mathbf{X}$ (thus $\eta$ is a $(\mathbf{K}, \mathbf{U})$ approximation, of $\mathbf{X}$, as well). Let $Y$ be a minimal subset of $X$ with the property that, for any $x \in X$, $\mathbf{K} \cap \mathbf{V}[\eta(x)] \neq \emptyset$ if and only if $\eta(x)=\eta(y)$ for some $y \in Y$. Then, necessarily, $\eta$ is injective on $Y$. There is an open entourage $\mathbf{W} \in \mathcal{U}, \mathbf{W} \subseteq \mathbf{V}$ such that the sets $\mathbf{W}[\eta(y)]$ $\mathbf{W}[\eta(z)]$ are disjoint for any $y \neq z$ in $Y$. Since all the sets $\mathbf{V}[\boldsymbol{x}], \mathbf{W}[\boldsymbol{x}]$ for $\boldsymbol{x} \in \mathbf{X}$ are open in $\mathbf{X}$, ordering the set $Y=\left\{y_{1}, \ldots, y_{m}\right\}$ and putting 


$$
\begin{aligned}
& \mathbf{S}_{1}=\mathbf{V}\left[\eta\left(y_{1}\right)\right] \backslash \bigcup_{j=2}^{m} \mathbf{W}\left[\eta\left(y_{j}\right)\right] \\
& \left.\mathbf{S}_{k}=\mathbf{V}\left[\eta\left(y_{k}\right)\right] \backslash \bigcup_{i=1}^{k-1} \mathbf{S}_{i} \cup \bigcup_{j=k+1}^{m} \mathbf{W}\left[\eta\left(y_{j}\right)\right]\right) \text { for } 1<k<m, \\
& \mathbf{S}_{m}=\mathbf{V}\left[\eta\left(y_{m}\right)\right] \backslash \bigcup_{i=1}^{m-1} \mathbf{S}_{i}
\end{aligned}
$$

we obtain a family of pairwise disjoint Borel sets $\left\{\mathbf{S}_{1}, \ldots, \mathbf{S}_{m}\right\}$ such that $\eta\left(y_{k}\right) \in \mathbf{S}_{k}$ for each $k \leq m$ and $\mathbf{K} \subseteq \mathbf{S}_{1} \cup \ldots \cup \mathbf{S}_{m}$. Let $\left\{x_{1}, \ldots, x_{n}\right\}=\left\{y_{k_{1}}, \ldots, y_{k_{n}}\right\}$ where $1 \leq k_{1}<\ldots<k_{n} \leq m$ are all those subscripts $k \leq m$ which satisfy $\mathbf{K} \cap \mathbf{S}_{k} \neq \emptyset$. Finally we put

$$
\mathbf{P}_{i}=\mathbf{K} \cap \mathbf{S}_{k_{i}}
$$

where $x_{i}=y_{k_{i}}$ for $i \leq n$. It is clear that $\boldsymbol{\pi}=\left\{\left(\mathbf{P}_{1}, x_{1}\right), \ldots,\left(\mathbf{P}_{n}, x_{n}\right)\right\}$ is a tagged Borel $(\mathbf{U}, \eta)$ partition of $\mathbf{K}$ we have been looking for. (The function $\sigma: \mathbf{K} \rightarrow X$ used in the proofs of Proposition 1.2.1 as well as of [19, Proposition 1.2.6] can be defined by $\sigma(\boldsymbol{x})=x_{i}$ for $\boldsymbol{x} \in \mathbf{P}_{i}$.)

Let $\boldsymbol{\mu}$ be a complex regular Borel measure on $\mathbf{X}$ with finite total variation. Depending on some finite (K, $\mathbf{U})$ approximation $\eta: X \rightarrow \mathbf{X}$ and a tagged Borel $(\mathbf{U}, \eta)$ partition $\boldsymbol{\pi}=\left\{\left(\mathbf{P}_{1}, x_{1}\right), \ldots,\left(\mathbf{P}_{n}, x_{n}\right)\right\}$ of $\mathbf{K}$, we will define a function $g_{\boldsymbol{\mu}}^{\boldsymbol{\pi}}: X \rightarrow \mathbb{C}$ enabling to approximate the integration with respect to the measure $\boldsymbol{\mu}$ in a sense to be made precise shortly. We denote $P_{i}=\eta^{-1}\left[\eta\left(x_{i}\right)\right]=\left\{x \in X: \eta(x)=\eta\left(x_{i}\right)\right\}$ for $i \leq n$ and put:

$$
g_{\boldsymbol{\mu}}^{\boldsymbol{\pi}}(x)= \begin{cases}\frac{\boldsymbol{\mu}\left(\mathbf{P}_{i}\right)}{\left|P_{i}\right|} & \text { for } x \in P_{i} \\ 0, & \text { for } x \in X \backslash \bigcup_{i=1}^{n} P_{i}\end{cases}
$$

Then the function $g_{\boldsymbol{\mu}}^{\pi}$ is an "approximate weak lifting" of $\boldsymbol{\mu}$, in the following sense.

Proposition 2.4.2 Let $\boldsymbol{\mu} \in \mathrm{M}(\mathbf{X}), \boldsymbol{h} \in \mathrm{C}_{\mathrm{b}}(\mathbf{X})$ and $\varepsilon>0$. Assume that $(\mathbf{K}, \mathbf{U})$ is an $\mathbf{X}$-raster such that $|\boldsymbol{\mu}|(\mathbf{X} \backslash \mathbf{K}) \leq \varepsilon$ and $|\boldsymbol{h}(\boldsymbol{x})-\boldsymbol{h}(\boldsymbol{y})| \leq \varepsilon$ for any $\boldsymbol{x}, \boldsymbol{y} \in \mathbf{X}$ whenever $\boldsymbol{x} \in \mathbf{K}$ and $(\boldsymbol{x}, \boldsymbol{y}) \in \mathbf{U}$. Finally, let $\eta: X \rightarrow \mathbf{X}$ be a finite $(\mathbf{K}, \mathbf{U})$ approximation of $\mathbf{X}$ and $\boldsymbol{\pi}=\left\{\left(\mathbf{P}_{1}, x_{1}\right), \ldots,\left(\mathbf{P}_{n}, x_{n}\right)\right\}$ be a tagged Borel $(\mathbf{U}, \eta)$ partition of $\mathbf{K}$. Then:

$$
\left|\int \boldsymbol{h} \mathrm{d} \boldsymbol{\mu}-\sum_{x \in X} \boldsymbol{h}(\eta x) g_{\boldsymbol{\mu}}^{\boldsymbol{\pi}}(x)\right| \leq \varepsilon\left(\|\boldsymbol{h}\|_{\infty}+\|\boldsymbol{\mu}\|\right)
$$


Before passing to the proof itself, the reader should realize that, since the variation $\|\boldsymbol{\mu}\|=|\boldsymbol{\mu}|(\mathbf{X})$ is finite, there is indeed a compact set $\mathbf{K} \subseteq \mathbf{X}$ such that $|\boldsymbol{\mu}|(\mathbf{X} \backslash \mathbf{K}) \leq \varepsilon$, and, due to the continuity of $\boldsymbol{h}$, the compactness of $\mathbf{K}$ and local compactness of $\mathbf{X}$, there is an entourage $\mathbf{U}$ such that $|\boldsymbol{h}(\boldsymbol{x})-\boldsymbol{h}(\boldsymbol{y})| \leq \varepsilon$ for any $\boldsymbol{x} \in \mathbf{K}, \boldsymbol{y} \in \mathbf{U}[\boldsymbol{x}]$.

Proof A straightforward computation using the notation from the definition of the function $g_{\boldsymbol{\mu}}^{\boldsymbol{\pi}}$ gives:

$$
\left|\int \boldsymbol{h} \mathrm{d} \boldsymbol{\mu}-\sum_{x \in X} \boldsymbol{h}(\eta x) g_{\boldsymbol{\mu}}^{\boldsymbol{\pi}}(x)\right| \leq\left|\int_{\mathbf{X} \backslash \mathbf{K}} \boldsymbol{h} \mathrm{d} \boldsymbol{\mu}\right|+\sum_{i=1}^{n}\left|\int_{\mathbf{P}_{i}} \boldsymbol{h} \mathrm{d} \boldsymbol{\mu}-\sum_{x \in P_{i}} \boldsymbol{h}(\eta x) g_{\boldsymbol{\mu}}^{\boldsymbol{\pi}}(x)\right|
$$

For the first summand on the right we have:

$$
\left|\int_{\mathbf{X} \backslash \mathbf{K}} \boldsymbol{h} \mathrm{d} \boldsymbol{\mu}\right| \leq \varepsilon\|\boldsymbol{h}\|_{\infty}
$$

Using the fact that $\eta(x)=\eta\left(x_{i}\right)$ for $x \in P_{i}$, we obtain the following estimate for each of the remaining right-hand summands:

$$
\begin{aligned}
\left|\int_{\mathbf{P}_{i}} \boldsymbol{h} \mathrm{d} \boldsymbol{\mu}-\sum_{x \in P_{i}} \boldsymbol{h}(\eta x) g_{\boldsymbol{\mu}}^{\boldsymbol{\pi}}(x)\right| & =\left|\int_{\mathbf{P}_{i}} \boldsymbol{h} \mathrm{d} \boldsymbol{\mu}-\boldsymbol{h}\left(\eta x_{i}\right) \boldsymbol{\mu}\left(\mathbf{P}_{i}\right)\right| \\
& =\left|\int_{\mathbf{P}_{i}}\left(\boldsymbol{h}-\boldsymbol{h}\left(\eta x_{i}\right)\right) \mathrm{d} \boldsymbol{\mu}\right| \leq \varepsilon\left|\boldsymbol{\mu}\left(\mathbf{P}_{i}\right)\right|
\end{aligned}
$$

Putting things together we obtain:

$$
\left|\int \boldsymbol{h} \mathrm{d} \boldsymbol{\mu}-\sum_{x \in X} \boldsymbol{h}(\eta x) g_{\boldsymbol{\mu}}^{\boldsymbol{\pi}}(x)\right| \leq \varepsilon\|\boldsymbol{h}\|_{\infty}+\varepsilon \sum_{i=1}^{n}\left|\boldsymbol{\mu}\left(\mathbf{P}_{i}\right)\right| \leq \varepsilon\left(\|\boldsymbol{h}\|_{\infty}+\|\boldsymbol{\mu}\|\right)
$$

In the special case when $\mathbf{X}=\mathbf{G}$ is a Hausdorff LCA group and $\boldsymbol{h}=\bar{\chi}$ where $\chi \in \widehat{\mathbf{G}}$ is a continuous character of $\mathbf{G}$ we obtain the following standard counterpart of Proposition 2.3.4 as a corollary. In the next three results we assume that the inner product on the unitary space $\mathbb{C}^{G}$ of functions on the finite abelian group $G$ below is normalized by the coefficient $d=1$.

Corollary 2.4.3 Let $\mathbf{G}$ be a Hausdorff LCA group, $\boldsymbol{\mu} \in \mathrm{M}(\mathbf{G}), \chi \in \widehat{\mathbf{G}}$ and $\varepsilon \in$ $(0,2 \pi / 3)$. Assume that $(\mathbf{K}, \mathbf{U})$ is a $\mathbf{G}$-raster such that $|\boldsymbol{\mu}|(\mathbf{X} \backslash \mathbf{K}) \leq \varepsilon$, and $\chi \in$ $\operatorname{Bohr}_{\varepsilon}(\mathbf{U})$. Finally, let $\eta: G \rightarrow \mathbf{G}$ be a finite $(\mathbf{K}, \mathbf{U})$ approximation of $\mathbf{G}$ and $\boldsymbol{\pi}=\left\{\left(\mathbf{P}_{1}, x_{1}\right), \ldots,\left(\mathbf{P}_{n}, x_{n}\right)\right\}$ be a tagged Borel $(\mathbf{U}, \eta)$ partition of $\mathbf{K}$. Then:

$$
\left|\mathbf{F}(\boldsymbol{\mu})(\chi)-\mathcal{F}_{\eta}\left(g_{\boldsymbol{\mu}}^{\pi}\right)(\chi)\right| \leq \varepsilon(1+\|\boldsymbol{\mu}\|)
$$


Proof It suffices to realize that $\widehat{\mathbf{G}} \subseteq \mathrm{C}_{\mathrm{b}}(\mathbf{G}),\|\chi\|_{\infty}=1$ for any $\chi \in \widehat{\mathbf{G}}$,

$$
|\chi(x)-\chi(y)| \leq 2 \sin \frac{\varepsilon}{2}<\varepsilon
$$

whenever $\boldsymbol{\chi} \in \operatorname{Bohr}_{\varepsilon}(\mathbf{U})$ and $\boldsymbol{x}, \boldsymbol{y} \in \mathbf{G}$ satisfy $\boldsymbol{x}-\boldsymbol{y} \in \mathbf{U}$, and, finally:

$$
\mathcal{F}_{\eta}\left(g_{\boldsymbol{\mu}}^{\boldsymbol{\pi}}\right)(\chi)=\left\langle g_{\boldsymbol{\mu}}^{\boldsymbol{\pi}}, \chi \circ \eta\right\rangle=\sum_{x \in G} g_{\boldsymbol{\mu}}^{\boldsymbol{\pi}}(x) \bar{\chi}(\eta x)
$$

The disadvantage of the modified Fourier transform $\mathcal{F}_{\eta}: \mathbb{C}^{G} \rightarrow \mathbb{C}^{\widehat{\mathbf{G}}}$ lies in the fact that, for $\chi \in \widehat{\mathbf{G}}$, the functions $\chi \circ \eta: G \rightarrow \mathbb{C}$ occurring in the expressions $\mathcal{F}_{\eta}(g)(\chi)=\langle g, \chi \circ \eta\rangle$ are, in general, just "approximate characters" and not true characters of the finite abelian group $G$. Thus the Fast Fourier Transform algorithm does not apply to the computation of the values $\mathcal{F}_{\eta}(g)(\chi)$ for $\chi \in \widehat{\mathbf{G}}$. This trouble can be overcome by making use of a "sufficiently good" adjoint pair of finite approximations $\eta: G \rightarrow \mathbf{G}, \phi: \widehat{G} \rightarrow \widehat{\mathbf{G}}$ of the LCA groups $\mathbf{G}, \widehat{\mathbf{G}}$, respectively; then each of the "approximate characters" $\chi \circ \eta$ of $G$ can be replaced by a genuine character $\gamma \in \widehat{G}$ such that $\phi(\gamma)$ is "close enough" to $\chi$. Nonetheless, the modified Fourier transform $\mathcal{F}_{\eta}$ will prove as a helpful auxiliary tool in deriving the needed estimates.

According to the Adjoint Approximation Scheme Theorem 2.5.5 from Zlatoš [19], for every $\mathbf{G}$-raster $(\mathbf{Q}, \mathbf{V})$, every $\widehat{\mathbf{G}}-\operatorname{raster}(\boldsymbol{\Delta}, \boldsymbol{\Upsilon})$ and any $\alpha=\varepsilon \in(0, \pi / 3)$, there exists an $\varepsilon$-adjoint $\varepsilon$-pairing preserving with reserve approximation scheme of the pair of groups $\mathbf{G}, \widehat{\mathbf{G}}$, given by some rasters $(\mathbf{K}, \mathbf{U}),(\boldsymbol{\Gamma}, \boldsymbol{\Omega})$, a finite abelian group $G$, a finite $(\mathbf{K}, \mathbf{U})$ approximation $\eta: G \rightarrow \mathbf{G}$ and a finite $(\boldsymbol{\Gamma}, \Omega)$ approximation $\phi: \widehat{G} \rightarrow \widehat{\mathbf{G}}$ of the groups $\mathbf{G}, \widehat{\mathbf{G}}$, respectively, compatible with the rasters $(\mathbf{Q}, \mathbf{V}),(\Delta, \Upsilon)$. All this means that $\mathbf{U} \subseteq \operatorname{Bohr}_{\varepsilon}(\boldsymbol{\Gamma}), \boldsymbol{\Omega} \subseteq \operatorname{Bohr}_{\varepsilon}(\mathbf{K})$,

$$
\left|\arg \frac{(\phi \gamma)(\eta x)}{\gamma(x)}\right| \leq \varepsilon
$$

for any $x \in G, \gamma \in \widehat{G}$ such that $\eta(x) \in \mathbf{K}+\mathbf{U}, \phi(\gamma) \in \Gamma \Omega$, as well as $(\mathbf{Q}, \mathbf{V}) \leq(\mathbf{K}, \mathbf{U})$, $(\Delta, \Upsilon) \leq(\boldsymbol{\Gamma}, \boldsymbol{\Omega})$ and:

$$
\operatorname{Bohr}_{\varepsilon}\left(\eta^{-1}[\mathbf{V}]\right) \subseteq \phi^{-1}[\boldsymbol{\Gamma}] \quad \operatorname{Bohr}_{\varepsilon}\left(\phi^{-1}[\mathbf{\Upsilon}]\right) \subseteq \eta^{-1}[\mathbf{K}]
$$

Moreover, one can arrange that at least one of the approximations $\eta, \phi$ is injective. However, neither the injectivity of $\eta$ or $\phi$ nor the last two inclusions will be used in the sequel. Additionally, given a complex regular Borel measure $\boldsymbol{\mu}$ on $\mathbf{G}$ with finite total variation, we can always choose $\mathbf{K}$ big enough to ensure that $|\boldsymbol{\mu}|(\mathbf{G} \backslash \mathbf{K}) \leq \varepsilon$. Again, $d>0$ denotes a normalizing multiplier for the inner product on the unitary space $\mathbb{C}^{G}$ of functions on the finite abelian group $G$ below. 
Theorem 2.4.4 (The Finite Fourier-Stieltjes Approximation Theorem) Let $\mathbf{G}$ be a Hausdorff LCA group, $\boldsymbol{\mu} \in \mathbf{M}(\mathbf{G})$ and $\varepsilon \in(0, \pi / 3)$. Assume that a $\mathbf{G}-$ raster $(\mathbf{K}, \mathbf{U})$ and a $\widehat{\mathbf{G}}$-raster $(\boldsymbol{\Gamma}, \boldsymbol{\Omega})$ together with a finite abelian group $G$ and maps $\eta: G \rightarrow \mathbf{G}$, $\phi: \widehat{G} \rightarrow \widehat{\mathbf{G}}$, form an $\varepsilon$-adjoint approximation scheme of the pair of groups $\mathbf{G}$, $\widehat{\mathbf{G}}$, which is $\varepsilon$-pairing preserving with reserve. Finally, let $|\boldsymbol{\mu}|(\mathbf{G} \backslash \mathbf{K}) \leq \varepsilon$ and $\boldsymbol{\pi}=\left\{\left(\mathbf{P}_{1}, x_{1}\right), \ldots,\left(\mathbf{P}_{n}, x_{n}\right)\right\}$ be a tagged Borel $(\mathbf{U}, \eta)$ partition of $\mathbf{K}$. Then

$$
\left|\mathbf{F}(\boldsymbol{\mu})(\chi)-\mathcal{F}\left(g_{\boldsymbol{\mu}}^{\pi}\right)(\gamma)\right| \leq \varepsilon(1+3\|\boldsymbol{\mu}\|)
$$

for any $\chi \in \Gamma, \gamma \in G$ such that $\phi(\gamma) \in \chi \Omega$.

Proof Under the conditions of the theorem we have:

$$
\left|\mathbf{F}(\boldsymbol{\mu})(\chi)-\mathcal{F}\left(g_{\boldsymbol{\mu}}^{\pi}\right)(\gamma)\right| \leq\left|\mathbf{F}(\boldsymbol{\mu})(\chi)-\mathcal{F}_{\eta}\left(g_{\boldsymbol{\mu}}^{\pi}\right)(\chi)\right|+\left|\mathcal{F}_{\eta}\left(g_{\boldsymbol{\mu}}^{\boldsymbol{\pi}}\right)(\chi)-\mathcal{F}\left(g_{\boldsymbol{\mu}}^{\boldsymbol{\pi}}\right)(\gamma)\right|
$$

Since $\chi \in \Gamma \subseteq \operatorname{Bohr}_{\varepsilon}(\mathbf{U})$, Corollary 2.4.3 implies that:

$$
\left|\mathbf{F}(\boldsymbol{\mu})(\chi)-\mathcal{F}_{\eta}\left(g_{\boldsymbol{\mu}}^{\boldsymbol{\pi}}\right)(\chi)\right| \leq \varepsilon(1+\|\boldsymbol{\mu}\|)
$$

Defining the sets $P_{i} \subseteq X$ as previously and denoting $P=P_{1} \cup \ldots \cup P_{n}$, we have supp $g \subseteq P$, implying the following estimate for the second summand on the right:

since

$$
\begin{aligned}
\left|\mathcal{F}_{\eta}\left(g_{\boldsymbol{\mu}}^{\boldsymbol{\pi}}\right)(\chi)-\mathcal{F}\left(g_{\boldsymbol{\mu}}^{\boldsymbol{\pi}}\right)(\gamma)\right| & =\left|\left\langle g_{\boldsymbol{\mu}}^{\boldsymbol{\pi}}, \chi \circ \eta\right\rangle-\left\langle g_{\boldsymbol{\mu}}^{\boldsymbol{\pi}}, \gamma\right\rangle\right|=\left|\left\langle g_{\boldsymbol{\mu}}^{\boldsymbol{\pi}},(\chi \circ \eta)-\gamma\right\rangle\right| \\
& \leq\left\|g_{\boldsymbol{\mu}}^{\boldsymbol{\pi}}\right\|_{1}\left\|((\chi \circ \eta)-\gamma) \cdot 1_{K}\right\|_{\infty} \\
& \leq|\boldsymbol{\mu}|(\mathbf{K}) \max _{x \in K}|\chi(\eta x)-\gamma(x)| \\
\left\|g_{\boldsymbol{\mu}}^{\boldsymbol{\pi}}\right\|_{1} & =\sum_{x \in X}\left|g_{\boldsymbol{\mu}}^{\boldsymbol{\pi}}(x)\right|=\sum_{i=1}^{n}\left|\boldsymbol{\mu}\left(\mathbf{P}_{i}\right)\right| \leq|\boldsymbol{\mu}|(\mathbf{K})
\end{aligned}
$$

If $x \in P, \chi \in \Gamma$ and $\phi(\gamma) \in \chi \Omega$ then $\eta(x) \in \mathbf{K}+\mathbf{U}, \chi^{-1} \phi(\gamma) \in \mathbf{\Omega} \subseteq \operatorname{Bohr}_{\varepsilon}(\mathbf{K})$ and $\phi(\gamma) \in \Gamma \Omega$, hence, due to the $\varepsilon$-pairing preservation with reserve:

$$
\begin{aligned}
|\chi(\eta x)-\gamma(x)| & \leq|\chi(\eta x)-(\phi \gamma)(\eta x)|+|(\phi \gamma)(\eta x)-\gamma(x)| \\
& \leq 2 \sin \frac{\varepsilon}{2}+2 \sin \frac{\varepsilon}{2}<2 \varepsilon
\end{aligned}
$$

Thus, finally:

$$
\left|\mathbf{F}(\boldsymbol{\mu})(\chi)-\mathcal{F}\left(g_{\boldsymbol{\mu}}^{\boldsymbol{\pi}}\right)(\gamma)\right| \leq \varepsilon(1+\| \boldsymbol{\mu} \mid)+2 \varepsilon|\boldsymbol{\mu}|(\mathbf{K}) \leq \varepsilon(1+3\|\boldsymbol{\mu}\|)
$$

It is clear that, for any $\boldsymbol{\mu} \in \mathbf{M}(\mathbf{G})$, the expression $\varepsilon(1+3\|\boldsymbol{\mu}\|)$ can be made arbitrarily small by choosing $\varepsilon$ small enough. It follows that, since the Fourier-Stieltjes transform $\widehat{\boldsymbol{\mu}}=\mathbf{F}(\boldsymbol{\mu}): \widehat{\mathbf{G}} \rightarrow \mathbb{C}$ is uniformly continuous, the discrete Fourier transform $\widehat{g}=\mathcal{F}(g)$ restricted to the set $\phi^{-1}[\Gamma \Omega] \subseteq G$ can serve as an arbitrarily precise table of the 
function $\widehat{\boldsymbol{\mu}}$ restricted to the set $\boldsymbol{\Gamma} \subseteq \widehat{\mathbf{G}}$, depending on the corresponding approximation scheme, $\|\boldsymbol{\mu}\|$ and $\varepsilon$.

Let $\boldsymbol{m}$ be the Haar measure on the Hausdorff LCA group $\mathbf{G}$; the inner product, the $p$-norms and the Fourier transform $\widehat{f}=\mathbf{F}(\boldsymbol{f})$ on the corresponding Lebesgue spaces $\mathrm{L}^{p}(\mathbf{G})(1 \leq p \leq 2)$ are defined via the Haar integral on $\mathbf{G}$. In the next theorem, nonetheless, the inner product, the corresponding norms and the discrete Fourier transform $\widehat{f}=\mathcal{F}(f)$ on the space $\mathbb{C}^{G}$ over a finite abelian group $G$ still use the normalizing multiplier $d=1$.

For a function $f \in \mathrm{L}^{1}(\mathbf{G})$ we denote by $\boldsymbol{\mu}_{f}$ the regular complex Borel measure such that $\mathrm{d} \boldsymbol{\mu}_{\boldsymbol{f}}=\boldsymbol{f} \mathrm{d} \boldsymbol{m}$; its total variation is $\left\|\boldsymbol{\mu}_{\boldsymbol{f}}\right\|=\|\boldsymbol{f}\|_{1}$. Given a $\mathbf{G}-\operatorname{raster}(\mathbf{K}, \mathbf{U})$, a finite $(\mathbf{K}, \mathbf{U})$ approximation $\eta: G \rightarrow \mathbf{G}$ of $\mathbf{G}$ and a tagged Borel $(\mathbf{U}, \eta)$ partition $\boldsymbol{\pi}=\left\{\left(\mathbf{P}_{1}, x_{1}\right), \ldots,\left(\mathbf{P}_{n}, x_{n}\right)\right\}$ of $\mathbf{K}$, we denote

$$
f^{\boldsymbol{\pi}}=g_{\boldsymbol{\mu}_{f}}^{\boldsymbol{\pi}}
$$

where the function $g_{\mu_{f}}^{\pi}$ was defined prior to Proposition 2.4.2. Then, as a special case of the Finite Fourier-Stieltjes Approximation Theorem 2.4.4, we obtain the following result.

Theorem 2.4.5 (The Finite Fourier Approximation Theorem 1) Let $\mathbf{G}$ be a Hausdorff LCA group, $\boldsymbol{f} \in \mathrm{L}^{1}(\mathbf{G})$ and $\varepsilon \in(0, \pi / 3)$. Assume that $\mathbf{G}-\operatorname{raster}(\mathbf{K}, \mathbf{U})$ and a $\widehat{\mathbf{G}}$-raster $(\boldsymbol{\Gamma}, \boldsymbol{\Omega})$ together with a finite abelian group $G$ and maps $\eta: G \rightarrow \mathbf{G}, \phi: \widehat{G} \rightarrow \widehat{\mathbf{G}}$ form an $\varepsilon$-adjoint approximation scheme of the pair of groups $\mathbf{G}, \widehat{\mathbf{G}}$ which is $\varepsilon$-pairing preserving with reserve. Finally, let $\left\|\boldsymbol{f} \cdot 1_{\mathbf{G} \backslash \mathbf{K}}\right\|_{1} \leq \varepsilon$ and $\boldsymbol{\pi}=\left\{\left(\mathbf{P}_{1}, x_{1}\right), \ldots,\left(\mathbf{P}_{n}, x_{n}\right)\right\}$ be a tagged Borel $(\mathbf{U}, \eta)$ partition of $\mathbf{K}$. Then

$$
\left|\mathbf{F}(\boldsymbol{f})(\chi)-\mathcal{F}\left(f^{\pi}\right)(\gamma)\right| \leq \varepsilon\left(1+3\|\boldsymbol{f}\|_{1}\right)
$$

for any $\chi \in \Gamma, \gamma \in G$ such that $\phi(\gamma) \in \chi \Omega$.

Since $\mathrm{C}_{\mathrm{c}}(\mathbf{G})$ is a dense subspace in $\mathrm{L}^{1}(\mathbf{G})$, it would be sufficient in some sense to deal with compactly supported continuous functions $f \in \mathrm{L}^{1}(\mathbf{G})$ in the last Theorem 2.4.5. In that case the composition $f=f \circ \eta: G \rightarrow \mathbb{C}$ can be taken as an approximate lifting of $\boldsymbol{f}$. As the Fourier transform $\widehat{\boldsymbol{f}}=\mathbf{F}(\boldsymbol{f}): \widehat{\mathbf{G}} \rightarrow \mathbb{C}$ of any function $\boldsymbol{f} \in \mathrm{L}^{1}(\mathbf{G})$ belongs to $\mathrm{C}_{0}(\widehat{\mathbf{G}})$, the composition $\widehat{\boldsymbol{f}} \circ \phi$ forms an approximate lifting of $\widehat{\boldsymbol{f}}$. If $\operatorname{supp} \boldsymbol{f} \subseteq \mathbf{K}$ then the function $f=\boldsymbol{f} \circ \eta$ restricted to the set $\eta^{-1}[\mathbf{K}]$ can serve as a sufficiently representative table of the function $f$ restricted to the set $\mathbf{K}$. The discrete Fourier transform $\widehat{f}=\mathcal{F}(f)=\mathcal{F}(\boldsymbol{f} \circ \eta)$ is close to the composition $\widehat{\boldsymbol{f}} \circ \phi$ on the set $\phi^{-1}[\boldsymbol{\Gamma}]$. Then both $\widehat{f}, \widehat{\boldsymbol{f}} \circ \phi$ restricted to the set $\phi^{-1}[\boldsymbol{\Gamma}]$ can serve as sufficiently representative 
tables of the function $\widehat{f}$ restricted to the set $\Gamma$. Before passing to a more precise formulation, we need to introduce some notions and formulate some preliminary results.

Given a Hausdorff LCA group $\mathbf{G}$, a symmetric compact neighborhood $\mathbf{K}$ of $0 \in \mathbf{G}$ and an $\varepsilon>0$, a pair $(\mathbf{U}, \eta)$ consisting of a symmetric neighborhood $\mathbf{U} \subseteq \mathbf{K}$ of 0 and a finite $(\mathbf{K}, \mathbf{U})$ approximation $\eta: G \rightarrow \mathbf{G}$ of $\mathbf{G}$ is called $\varepsilon$-adequate for $\mathbf{K}$ if

$$
\left|\int \boldsymbol{f} \mathrm{d} \boldsymbol{m}-\frac{\boldsymbol{m}(\mathbf{K})}{\left|\eta^{-1}[\mathbf{K}]\right|} \sum_{x \in G} \boldsymbol{f}(\eta x)\right| \leq \varepsilon\|\boldsymbol{f}\|_{\infty}
$$

for every continuous function $f: \mathbf{G} \rightarrow \mathbb{C}$ such that $\operatorname{supp} f \subseteq \mathbf{K}$.

Once we realize that every HFI approximation $\eta: G \rightarrow{ }^{*} \mathbf{G}$ is a $\left({ }^{*} \mathbf{K},{ }^{*} \mathbf{U}\right)$ approximation for any $\mathbf{G}$-raster $(\mathbf{K}, \mathbf{U})$ and, due to the fact that the Haar measure on $\mathbf{G}$ can be obtained by pushing down the Loeb measure on ${ }^{*} \mathbf{G}$ obtained from the normalizing multiplier $d=\boldsymbol{m}(\mathbf{K}) /\left|\eta^{-1}[\mathbf{K}]\right|$, the pair $(\mathbf{U}, \eta)$ is $\varepsilon$-adequate for $\mathbf{K}$ and each $\varepsilon>0$, we readily obtain the following lemma by the transfer principle.

Lemma 2.4.6 Let $\mathbf{G}$ be a Hausdorff LCA group, $\mathbf{K}$ be a symmetric compact neighborhood of $0 \in \mathbf{G}$ and $\varepsilon>0$. Then there exists a symmetric neighborhood $\mathbf{U}_{0} \subseteq \mathbf{K}$ of 0 such that for any $\mathbf{G}$-raster $(\mathbf{K}, \mathbf{U})$ satisfying $\mathbf{U} \subseteq \mathbf{U}_{0}$ and any finite $(\mathbf{K}, \mathbf{U})$ approximation $\eta: G \rightarrow \mathbf{G}$ the pair $(\mathbf{U}, \eta)$ is $\varepsilon$-adequate for $\mathbf{K}$.

In the next theorem the inner product on the finite dimensional linear space $\mathbb{C}^{G}$, the discrete Fourier transform $\mathcal{F}: \mathbb{C}^{G} \rightarrow \mathbb{C}^{\widehat{G}}$ and all the $p$-norms on $\mathbb{C}^{G}$ use the normalizing coefficient

$$
d=\frac{\boldsymbol{m}(\mathbf{K})}{|K|}
$$

where $K=\eta^{-1}[\mathbf{K}]$.

Theorem 2.4.7 (The Finite Fourier Approximation Theorem 2) Let $\mathbf{G}$ be a Hausdorff LCA group, $\boldsymbol{f} \in \mathrm{C}_{\mathrm{c}}(\mathbf{G})$ and $\varepsilon \in(0, \pi / 3)$. Assume that a $\mathbf{G}$-raster $(\mathbf{K}, \mathbf{U})$ and a $\widehat{\mathbf{G}}$-raster $(\boldsymbol{\Gamma}, \boldsymbol{\Omega})$ together with a finite abelian group $G$ and maps $\eta: G \rightarrow \mathbf{G}$, $\phi: \widehat{G} \rightarrow \widehat{\mathbf{G}}$ form an $\varepsilon$-adjoint approximation scheme of the pair of groups $\mathbf{G}, \widehat{\mathbf{G}}$ which is $\varepsilon$-pairing preserving with reserve. Finally, let supp $\boldsymbol{f} \subseteq \mathbf{K}$ and the pair $(\mathbf{U}, \eta)$ be $\varepsilon$-adequate for $\mathbf{K}$. Then

$$
|\mathbf{F}(\boldsymbol{f})(\chi)-\mathcal{F}(\boldsymbol{f} \circ \eta)(\gamma)| \leq \varepsilon(1+2 \boldsymbol{m}(\mathbf{K}))\|\boldsymbol{f}\|_{\infty}
$$

for any $\chi \in \Gamma, \gamma \in G$ such that $\phi(\gamma) \in \chi \Omega$. 
Proof Since

$|\mathbf{F}(\boldsymbol{f})(\chi)-\mathcal{F}(\boldsymbol{f} \circ \eta)(\gamma)| \leq\left|\mathbf{F}(\boldsymbol{f})(\chi)-\mathcal{F}_{\eta}(\boldsymbol{f} \circ \eta)(\chi)\right|+\left|\mathcal{F}_{\eta}(\boldsymbol{f} \circ \eta)(\chi)-\mathcal{F}(\boldsymbol{f} \circ \eta)(\gamma)\right|$

it suffices to estimate the summands on the right. As $\operatorname{supp}(f \cdot \bar{\chi})=\operatorname{supp} f \subseteq \mathbf{K}$ and $|\chi(\boldsymbol{x})|=1$ for each $\boldsymbol{x} \in \mathbf{G}$, under the assumptions of the theorem we have

$$
\begin{aligned}
\left|\mathbf{F}(\boldsymbol{f})(\chi)-\mathcal{F}_{\eta}(\boldsymbol{f} \circ \eta)(\chi)\right| & =\left|\int \boldsymbol{f} \bar{\chi} \mathrm{d} \boldsymbol{m}-d \sum_{x \in G} \boldsymbol{f}(\eta x) \bar{\chi}(\eta x)\right| \\
& \leq \varepsilon\|\boldsymbol{f} \cdot \bar{\chi}\|_{\infty}=\varepsilon\|\boldsymbol{f}\|_{\infty}
\end{aligned}
$$

as well as:

$$
\begin{aligned}
\left|\mathcal{F}_{\eta}(\boldsymbol{f} \circ \eta)(\chi)-\mathcal{F}(\boldsymbol{f} \circ \eta)(\gamma)\right| & =\left|\left\langle\boldsymbol{f} \circ \eta,((\chi \circ \eta)-\gamma) \cdot 1_{K}\right\rangle\right| \\
& \left.\leq\|\boldsymbol{f} \circ \eta\|_{1} \|((\chi \circ \eta)-\gamma)\right) \cdot 1_{K} \|_{\infty}
\end{aligned}
$$

Then

$$
\|\boldsymbol{f} \circ \eta\|_{1}=d \sum_{x \in K}|\boldsymbol{f}(\eta x)| \leq d|K|\|\boldsymbol{f} \circ \eta\|_{\infty} \leq \boldsymbol{m}(\mathbf{K})\|\boldsymbol{f}\|_{\infty}
$$

and

$$
|\chi(\eta x)-\gamma(x)| \leq|\chi(\eta x)-(\phi \gamma)(\eta x)|+|(\phi \gamma)(\eta x)-\gamma(x)| \leq 2 \sin \frac{\varepsilon}{2}+2 \sin \frac{\varepsilon}{2} \leq 2 \varepsilon
$$

whenever $\phi(\gamma) \in \chi \Omega$ and $x \in K=\eta^{-1}[\mathbf{K}]$. The second inequality of the theorem follows immediately.

In particular, for $\chi=\phi(\gamma)$ a brief inspection of the proof gives the estimate

$$
|\mathbf{F}(\boldsymbol{f})(\phi \gamma)-\mathcal{F}(\boldsymbol{f} \circ \eta)(\gamma)| \leq \varepsilon(1+\boldsymbol{m}(\mathbf{K}))\|\boldsymbol{f}\|_{\infty}
$$

for $\gamma \in \phi^{-1}[\boldsymbol{\Gamma}]$ under the conditions of the last theorem (and even without the assumption that the approximation scheme is $\varepsilon$-preserving with reserve). This result can be generalized to all the Fourier transforms $\mathbf{F}: \mathrm{L}^{p}(\mathbf{G}) \rightarrow \mathrm{L}^{q}(\widehat{\mathbf{G}})$ for any pair of adjoint exponents $p \in[1,2], q \in[2, \infty]$, in particular, to the Fourier-Plancherel transform $\mathbf{F}: \mathrm{L}^{2}(\mathbf{G}) \rightarrow \mathrm{L}^{2}(\widehat{\mathbf{G}})$.

In what follows the $q$-norms on the linear space $\mathbb{C}^{\widehat{G}}$ are normalized by means of the dual normalizing coefficient

$$
\widehat{d}=\frac{1}{d|G|}=\frac{|K|}{\boldsymbol{m}(\mathbf{K})|G|}
$$


Theorem 2.4.8 (The Generalized Finite Fourier Approximation Theorem) Let $\mathbf{G}$ be a Hausdorff LCA group, $\boldsymbol{f} \in \mathrm{C}_{\mathrm{c}}(\mathbf{G}), 2 \leq q \leq \infty$ and $\varepsilon \in(0, \pi / 3)$. Assume that a $\mathbf{G}-\operatorname{raster}(\mathbf{K}, \mathbf{U})$ and a $\widehat{\mathbf{G}}-$ raster $(\boldsymbol{\Gamma}, \boldsymbol{\Omega})$ together with a finite abelian group $G$ and maps $\eta: G \rightarrow \mathbf{G}, \phi: \widehat{G} \rightarrow \widehat{\mathbf{G}}$ form an $\varepsilon$-adjoint, $\varepsilon$-pairing preserving approximation scheme of the pair of groups $\mathbf{G}, \widehat{\mathbf{G}}$. Finally, let $\operatorname{supp} \boldsymbol{f} \subseteq \mathbf{K}$ and the pair $(\mathbf{U}, \eta)$ be $\varepsilon$-adequate for $\mathbf{K}$. Then, denoting $\Gamma=\phi^{-1}[\boldsymbol{\Gamma}]$, we have:

$$
\left\|((\mathbf{F}(\boldsymbol{f}) \circ \phi)-\mathcal{F}(\boldsymbol{f} \circ \eta)) \cdot 1_{\Gamma}\right\|_{q} \leq \varepsilon\left(\frac{|K||\Gamma|}{\boldsymbol{m}(\mathbf{K})|G|}\right)^{1 / q}(1+\boldsymbol{m}(\mathbf{K}))\|\boldsymbol{f}\|_{\infty}
$$

Proof As already mentioned prior to the theorem, the case $p=1, q=\infty$ (under the convention $1 / \infty=0$ ) directly follows from the proof of Theorem 2.4.7. Using this fact, a straightforward computation gives:

$$
\begin{aligned}
\left\|((\mathbf{F}(\boldsymbol{f}) \circ \phi)-\mathcal{F}(\boldsymbol{f} \circ \eta)) \cdot 1_{\Gamma}\right\|_{q} & \leq\left(\widehat{d} \sum_{\gamma \in \Gamma}|\mathbf{F}(\boldsymbol{f})(\phi \gamma)-\mathcal{F}(\boldsymbol{f} \circ \eta)(\gamma)|^{q}\right)^{1 / q} \\
& \leq(\widehat{d}|\Gamma|)^{1 / q} \varepsilon(1+\boldsymbol{m}(\mathbf{K}))\|\boldsymbol{f}\|_{\infty} \\
& \leq \varepsilon\left(\frac{|K||\Gamma|}{\boldsymbol{m}(\mathbf{K})|G|}\right)^{1 / q}(1+\boldsymbol{m}(\mathbf{K}))\|\boldsymbol{f}\|_{\infty}
\end{aligned}
$$

The function $\mathbf{F}(\boldsymbol{f}) \circ \phi$ restricted to the set $\Gamma \subseteq \widehat{G}$ can be viewed as the table of the Fourier transform $\widehat{\boldsymbol{f}}=\mathbf{F}(\boldsymbol{f})$ evaluated on the finite set of arguments $\phi(\gamma) \in \boldsymbol{\Gamma} \subseteq \widehat{\mathbf{G}}$. On the other hand, the function $f=f \circ \eta$ restricted to the set $K \subseteq G$ can be viewed as the table of the original function $f$ evaluated on the finite set of arguments $\eta(x) \in \mathbf{K} \subseteq \mathbf{G}$. Then the function $\widehat{f}=\mathcal{F}(f \circ \eta)$ is the discrete Fourier transform of $f$ computed on the basis of this table. Under the conditions of Theorem 2.4.8, the tables represented by the functions $\mathbf{F}(\boldsymbol{f}) \circ \phi, \mathcal{F}(\boldsymbol{f} \circ \eta)$ restricted to $\Gamma$ are close to each other in the sense of any of the norms $\|\cdot\|_{q}$ where $2 \leq q \leq \infty$. Moreover, the theorem gives some explicit upper bounds on how they can differ.

Theorems 2.4 .7 and 2.4.8 can easily be generalized, under slightly modified upper bounds, to functions $\boldsymbol{f} \in \mathrm{C}_{0}(\mathbf{G}) \cap \mathrm{L}^{1}(\mathbf{G})$ or $\boldsymbol{f} \in \mathrm{C}_{0}(\mathbf{G}) \cap \mathrm{L}^{p}(\mathbf{G})$. This is left to the reader. As pointed out by Gordon in [7] for the Fourier-Plancherel transform $\mathrm{L}^{2}(\mathbf{G}) \rightarrow \mathrm{L}^{2}(\widehat{\mathbf{G}})$, the class of functions $f \in \mathrm{L}^{2}(\mathbf{G})$ approximable by the composition $f \circ \eta$ such that their Fourier transform $\mathbf{F}(\boldsymbol{f})$ is simultaneously approximable by the discrete Fourier transform $\mathcal{F}(f \circ \eta)$ in the sense of Theorem 2.4.8 contains even more general functions, namely such that both $\boldsymbol{f}$ and $\widehat{\boldsymbol{f}}$ are Riemann integrable, ie, continuous almost everywhere with respect to the Haar measures on $\mathbf{G}, \widehat{\mathbf{G}}$, respectively. It is clear that Gordon's remark applies to any pair of adjoint exponents $p \in[1,2], q \in[2, \infty]$ and not just to the Hilbert space case $p=q=2$. 


\section{References}

[1] S Albeverio, J E Fenstad, R Høegh-Krohn, T Lindstrøm, Nonstandard Methods in Stochastic Analysis and Mathematical Physics, Academic Press, London-New York-Orlando (1986)

[2] S Albeverio, E I Gordon, A Y Khrennikov, Finite-dimensional approximations of operators in the Hilbert spaces of functions on locally compact abelian groups, Acta Appl. Math. 64 (2000) 33-73; https://doi.org/10.1023/A:1006457731833

[3] M Davis, Applied Nonstandard Analysis, John Wiley Sons, New York-London (1977)

[4] N Dinculeanu, On Kolmogorov-Tamarkin and M. Riesz compactness criteria in function spaces over a locally compact group, J. Math. Anal. Appl. 89 (1982) 67-85; https://doi.org/10.1016/0022-247X(82)90091-9

[5] CL Epstein, How well does the finite Fourier transform approximate the Fourier transform?, Communications Pure Appl. Math. 58 (2005) 1-15; https://doi.org/10.1002/ cpa. 20064

[6] E I Gordon, Nonstandard analysis and locally compact abelian groups, Acta Appl. Math. 25 (1991) 221-239; https://doi.org/10.1007/BF00047004

[7] E I Gordon, Nonstandard Methods in Commutative Harmonic Analysis, volume 164, Translations of Mathematical Monographs, Amer. Math. Soc., Providence, R. I. (1997); https://doi.org/10.1090/mmono/164

[8] K Gröchenig, Foundations of Time-Frequency Analysis, Birkhäuser, Boston-BaselBerlin (2001)

[9] C W Henson, L C Moore, Nonstandard analysis and the theory of Banach spaces, from: "Nonstandard Analysis, Recent Developments", (A E Hurd, editor), Springer, Berlin-Heidelberg-New York (1983) 27-112; https://doi.org/10.1007/BFb0065334

[10] E Hewitt, KA Ross, Abstract Harmonic Analysis I, Springer, Berlin-GötingenHeidelberg (1963); https://doi.org/10.1007/978-3-662-40409-6

[11] E Hewitt, K A Ross, Abstract Harmonic Analysis II, Springer, Berlin-Heidelberg-New York (1970); https://doi.org/10.1007/978-3-662-26755-4

[12] L H Loomis, An Introduction to Abstract Harmonic Analysis, D. Van Nostrand, Princeton, N. J. (1953)

[13] G K Pedersen, Analysis Now, 2 edition, Graduate Texts in Mathematics, Springer, Berlin-Heidelberg-New York (1995)

[14] R L Pego, Compactness in $L^{2}$ and the Fourier transform, Proc. Amer. Math. Soc. 92 (1985) 252-254; https://doi.org/10.1090/S0002-9939-1985-0801333-9

[15] W Rudin, Measure algebras on abelian groups, Bull. Amer. Math. Soc. 65 (1959) 227-247; https://doi.org/10.1090/S0002-9904-1959-10322-0 
[16] W Rudin, Fourier Analysis on Groups, Interscience, John Wiley and Sons, New York-London (1962)

[17] T Tao, Higher Order Fourier Analysis, Graduate Studies in Mathematics, vol. 142, Amer. Math. Soc., Providence, R. I. (2012)

[18] M Ziman, P Zlatoš, Hyperfinite dimensional representations of spaces and algebras of measures, Monatsh. Math. 148 (2006) 241-261; https://doi.org/10.1007/s00605-0050375-3

[19] P Zlatoš, Gordon's Conjectures 1 and 2: Pontryagin-van Kampen duality in hyperfinite setting, J. Logic \& Analysis 13 (2021) 1-71; https://doi.org/10.4115/jla.2021.13.1

Faculty of Mathematics, Physics and Informatics, Comenius University, 84248 Bratislava, Slovakia

zlatos@fmph.uniba.sk

Received: 11 May 2019 Revised: 1 April 2021 